\title{
TOPOLOGICAL FRAME EXTENSION
}

\author{
ZOHREH VAZIRY AND DIETER LESEBERG
}

\begin{abstract}
The concept of nearness on a set was introduced by H. Herrlich. D. Leseberg generalized nearness by introducing supernearness, which generalizes also supertopology as defined by D. Doitchinov. In this paper, our work is based on the representation theorem of $M$. H. Stone and the definition of nearness. We define proximity and nearness on a Boolean frame and then, by using these, we define supertopic frame, supernear frame and paranear frame. We study basic properties of the concepts defined. We also introduce a topological extension on a Boolean frame and investigate its behavior.
\end{abstract}

\section{INTRODUCTION}

Nearness spaces were introduced by H. Herrlich $[4,5]$ in 1974 as an axiomatization of the concept of nearness of arbitrary collection of sets. The supernearness spaces introduced by D. Leseberg [8] in 2002 are a natural generalization of the nearness spaces and the supertopological spaces as defined by D. Doitchinov [3]. Recall that, by the Stone representation theorem [13], for each Boolean algebra $B$ or, equivalently, Boolean ring $B$, there exist a set $X$ and an injective function $i: B \rightarrow$ $P X$ such that the following holds: $i(0)=\emptyset ; i(1)=X ; i(a \wedge b)=i(a) \cap i(b)$; $i(a \vee b)=i(a) \cup i(b)$ and $i\left(a^{\prime}\right)=X \backslash i(a)$. Hence, $B$ is isomorphic to the set-algebra on $i(B)$.

This paper is based on the Stone representation theorem and the definition of nearness by $\mathrm{H}$. Herrlich. We define proximity and nearness on Boolean frame and then, by using these, we define a supertopic frame, supernear frame and paranear frame. Then, we introduce a topological extension on a Boolean frame. Topological extensions play an important role in the theory of proximities or nearness. For example, Herrlich found a useful generalization of contiguity spaces by introducing nearness spaces, and Bentley [2] showed that those nearness spaces which can be extended to topological ones have a neat internal characterization.

D. Leseberg [9-11] introduced supernearness spaces and the corresponding topological extensions. Here we give a corresponding description in the realm of Boolean frames.

$M S C$ (2010): primary 54A05, 54D35, 54E05, 06C15, 06D22, 54E17; secondary 18B30, 18B35, 54B30.

Keywords: Boolean frame, near frame, supernear frame, paranear frame, extension, frame extension. 


\section{BACKGROUND}

Definition 2.1. ([7,14]) A complete lattice $L$ is called a frame if and only if it satisfies the following $1^{\text {st }}$ distributive law:

$(I F D 1) \forall a \in L, \forall S \subseteq L, a \wedge \bigvee S=\bigvee\{a \wedge x \mid x \in S\}$

A frame $L$ is called Boolean if and only if it is complementary. Note that, in this case, each element has a unique complement, i.e., $\forall a \in L \exists$ ! $a^{\prime} \in L$ s.t. $a \wedge a^{\prime}=0$ and $a \vee a^{\prime}=1$. Therefore, a Boolean frame additionally satisfies the following $2^{\text {nd }}$ distributive law:

$(I F D 2) \forall a \in L, \forall S \subseteq L, a \vee \bigwedge S=\bigwedge\{a \vee x \mid x \in S\}$

Frame homomorphisms between Boolean frames preserve top, bottom (denoted by 1 and 0 respectively), meets, joins and complements.

Definition 2.2. ([14]) Let $A$ and $B$ be subsets of a Boolean frame $L$. Then we define:

(1) $\sec A=\{x \in L \mid \forall a \in A, x \wedge a \neq 0\}$;

(2) $\mathfrak{s t a c k} A=\{x \in L \mid \exists a \in A$ s.t. $a \leq x\}$;

(3) $A \bigvee B=\{a \vee b \mid a \in A, b \in B\}$;

(4) $A \bigwedge B=\{a \wedge b \mid a \in A, b \in B\}$;

(5) $A^{\prime}=\left\{a^{\prime} \mid a \in A\right\}$;

(6) $\operatorname{st}(x, A)=\bigvee\{a \in A \mid a \wedge x \neq 0\}$;

(7) $\operatorname{st}(x, A)^{d}=\bigwedge\{a \in A \mid a \vee x \neq 1\}$;

(8) $A<<B$ iff $\forall a \in A \exists b \in B$ s.t. $b \leq a$ ( $A$ corefines $B)$;

(9) $A \prec B$ iff $\forall a \in A \exists b \in B$ s.t. $a \leq b$ ( $A$ refines $B$ ).

\section{Supertopic Frame AND SUPERneAr Frame}

Definition 3.1. Let $F$ be a subset of a Boolean frame. Then, $F$ is called a frame filter if and only if it satisfies the following conditions:

(ffi $) ~ F \neq \emptyset$;

(ffi $)_{2} a_{1}, a_{2} \in F$ if and only if $a_{1} \wedge a_{2} \in F$.

We denote the set of all frame filters in a Boolean frame $L$ by $\mathbf{F F I L ( L ) . ~}$

Definition 3.2. Let $G$ be a subset of a Boolean frame. Then, $G$ is called frame grill if and only if it satisfies the following conditions:

$\left(\right.$ fgri $\left._{1}\right) \quad 0 \notin G$;

$\left(\right.$ fgri $\left._{2}\right) a_{1} \in G$ or $a_{2} \in G$ if and only if $a_{1} \vee a_{2} \in G$.

We denote the set of all frame grills in a Boolean frame $L$ by $\mathbf{F G R L ( L ) . ~}$

Proposition 3.3. Let $A$ be a subset of a Boolean frame L. Then the following statements hold.

(1) If $A$ is a frame grill, then $\mathfrak{s e c} A$ is a frame filter.

(2) If $A$ is a frame filter, then $\mathfrak{s e c} A$ is a frame grill.

Proof. (1) First we show that the statement (1) holds. Let $A$ be a frame grill, so $0 \notin A$. If $A=\emptyset$, then $\mathfrak{s e c} A=L$, which is a filter. If $A \neq \emptyset$, then there exists $a(\neq 0) \in A$, so $1 \in \mathfrak{s e c} A$, therefore, $\mathfrak{s e c} A \neq \emptyset$. Suppose $b_{1}, b_{2} \in \mathfrak{s e c} A$. Then, for all $a \in A$, we have $b_{1} \wedge a \neq 0$ and $b_{2} \wedge a \neq 0$. Now we have to show that, for 
all $a \in A,\left(b_{1} \wedge b_{2}\right) \wedge a \neq 0$. Let $a$ be an arbitrary element of $A$. By considering $A$ is a frame grill and $a=\left(b_{2} \wedge a\right) \vee\left(b_{2}^{\prime} \wedge a\right)$, either $b_{2} \wedge a \in A$ or $b_{2}^{\prime} \wedge a \in A$. If $b_{2}^{\prime} \wedge a \in A$, then, since $b_{2} \in \mathfrak{s e c} A$, we get a contradiction. Therefore, $b_{2} \wedge a \in A$, which, by considering $b_{1} \in \mathfrak{s e c} A$, implies $b_{1} \wedge\left(b_{2} \wedge a\right) \neq 0$. So, for all $a \in A$, we have, $\left(b_{1} \wedge b_{2}\right) \wedge a \neq 0$, i.e., $b_{1} \wedge b_{2} \in \mathfrak{s e c} A$. Also, it is obvious that, if $b_{1} \wedge b_{2} \in \mathfrak{s e c} A$, then $b_{1} \in \mathfrak{s e c} A$ and $b_{2} \in \mathfrak{s e c} A$.

Now we show that the statement (2) holds. Let $A$ be a frame filter. Then $A \neq \emptyset$, therefore, $0 \notin \mathfrak{s e c} A$. If $0 \in A$, then $\mathfrak{s e c} A=\emptyset$, which is a frame grill. If $0 \notin A$, then there exists $(0 \neq) a \in A$, which implies $1 \in \mathfrak{s e c} A$, i.e., $\mathfrak{s e c} A \neq \emptyset$. Suppose $b_{1}$ and $b_{2}$ are elements of $L$ such that $b_{1} \vee b_{2} \in \mathfrak{s e c} A$. Then, for all $a \in A$, $\left(b_{1} \vee b_{2}\right) \wedge a \neq 0$, i.e., $\left(b_{1} \wedge a\right) \vee\left(b_{2} \wedge a\right) \neq 0$. So, for all $a \in A$, either $b_{1} \wedge a \neq 0$ or $b_{2} \wedge a \neq 0$. Suppose $a_{1}, a_{2} \in A$ such that $b_{2} \wedge a_{1}=0$ and $b_{1} \wedge a_{2}=0$. Then, by knowing $A$ is a frame filter, we have $a_{1} \wedge a_{2} \in A$. But, by our assumption, $\left(a_{1} \wedge a_{2}\right) \wedge b_{1}=0$ and $\left(a_{1} \wedge a_{2}\right) \wedge b_{2}=0$, which is a contradiction. So, either, for all $a \in A, b_{1} \wedge a \neq 0$ or, for all $a \in A, b_{2} \wedge a \neq 0$, i.e., either $b_{1} \in \mathfrak{s e c} A$ or $b_{2} \in \mathfrak{s e c} A$. Also, it is obvious that, if $b_{1} \in \mathfrak{s e c} A$ or $b_{2} \in \mathfrak{s e c} A$, then $b_{1} \vee b_{2} \in \mathfrak{s e c} A$.

Definition 3.4. Let $L$ be a Boolean frame. A function $-{ }^{c}: L \rightarrow L$ is called a frame-closure on $L$ if and only if it satisfies the following conditions:

$\left(\mathrm{fcl}_{1}\right) 0^{c}=0$

$\left(\mathrm{fcl}_{2}\right) x, z \in L$ and $x \leq z$ imply $x^{c} \leq z^{c}$;

$\left(\mathrm{fcl}_{3}\right) x \in L$ implies $x \leq x^{c}$

$\left(\mathrm{fcl}_{4}\right) x, z \in L$ implies $(x \vee z)^{c} \leq x^{c} \vee z^{c}$;

$\left(\mathrm{fcl}_{5}\right) x \in L$ implies $\left(x^{c}\right)^{c} \leq x^{c}$.

Remark 3.5. For every frame-closure $c$, we have $1^{c}=1$.

Example 3.6. Each Boolean frame $L$ has an underlying frame closure ${ }^{-L}$ defined by setting: $\overline{0}^{L}:=0, \overline{1}^{L}:=1$ and, for $x \in L \backslash\{0,1\}, \bar{x}^{L}:=\vee\{z \in L \backslash\{0,1\}$ : $\left.x^{\prime} \leq z^{\prime}\right\}$. Here we mention that ${ }^{-L}$ preserves an arbitrary join, i.e., $A \subset L$ implies $\vee \bar{A}^{L}=\overline{\mathrm{V} A}^{L}$.

Definition 3.7. Let $L$ be a Boolean frame with a frame-closure $c: L \rightarrow L$. Then $c$ is called a symmetrical frame closure if and only if it satisfies the following condition:

(sym) $x, z \in L \backslash\{0,1\}$ and $x \leq z^{c}$ imply $z \leq x^{c}$.

Definition 3.8. Let $L$ be a Boolean frame. A relation $\delta \subset L \times L$ is called a PE-proximity on $L$, and $(L, \delta)$ is called a PE-proximal frame if and only if the following hold:

$\left(\mathrm{pp}_{1}\right) a, b \in L$ and $a \bar{\delta} b$ imply $\bar{b}^{\delta} \leq a^{\prime}$, where $a \bar{\delta} b$ means $a$ is not in relation with $b$, $\overline{0}^{\delta}:=0, \overline{1}^{\delta}:=1$ and for each $c \in L \backslash\{0,1\}, \bar{c}^{\delta}:=\vee\{d \in L \backslash\{0,1\}: d \delta c\} ;$

$\left(\mathrm{pp}_{2}\right) a, b \in L$ implies $a \bar{\delta} 0$ and $0 \bar{\delta} b$;

$\left(\mathrm{pp}_{3}\right) a \delta(b \vee c)$ if and only if $a \delta b$ or $a \delta c$;

$\left(\mathrm{pp}_{4}\right) a \in L \backslash\{0\}$ implies $a \delta a$;

$\left(\mathrm{pp}_{5}\right) a \delta b$ and $a \leq c \in L$ imply $c \delta b$;

$\left(\mathrm{pp}_{6}\right) a \bar{\delta} b$ implies there exists $c \in L$ such that $a \bar{\delta} c^{\prime}$ and $c \bar{\delta} b$. 
Let $\left(L, \delta_{1}\right)$ and $\left(M, \delta_{2}\right)$ be two PE-proximal frames. Then, a frame homomorphism $f: L \rightarrow M$ is called proximal continuous if and only if it satisfies the following condition:

$$
x \delta_{1} y \text { implies } f(x) \delta_{2} f(y) .
$$

The corresponding category is denoted by PEProxFrm.

Definition 3.9. In the above definition, if we replace the axiom $\left(\mathrm{pp}_{6}\right)$ by the following axiom $\left(\operatorname{lp}_{6}\right)$, then we call $\delta$ a $L E$-proximity on $L$ and the pair $(L, \delta)$ an LE-proximal frame.

$\left(\operatorname{lp}_{6}\right) a \delta b$ and $b \leq \bar{c}^{\delta}$ imply $a \delta c$.

The corresponding category whose morphisms are the proximal continuous homomorphisms is denoted by LEProxFrm.

Definition 3.10. Let $(L, \delta)$ be a PE-proximal (or LE-proximal) frame. If it satisfies the following symmetric condition, then it is called an EF-proximal (respectively, LO-proximal) frame.

(sym) $a, b \in L$ and $a \delta b$ imply $b \delta a$,

The corresponding category is denoted by EFProxFrm (respectively, LOProxFrm).

Remark 3.11. Let $(L, \delta)$ be a PE-proximal (or LE-proximal) frame. Then, the following holds:

$$
a, b \in L \text { and } a \wedge b \neq 0 \text { imply } a \delta b .
$$

Proof. We consider the properties of a PE-proximal (or LE-proximal) frame. By $\left(\mathrm{pp}_{4}\right)$, we have, $(a \wedge b) \delta(a \wedge b)$ and, by using $\left(\mathrm{pp}_{5}\right)$, we can say $a \delta(a \wedge b)$. Now by $\left(\mathrm{pp}_{3}\right)$ we have $a \delta((a \wedge b) \vee b)$, i.e., $a \delta b$.

Remark 3.12. Every PE-proximal frame is an LE-proximal frame.

Proof. Let $(L, \delta)$ be a PE-proximal frame. Then we have to show that $\left(\operatorname{lp}_{6}\right)$ holds, which means $(L, \delta)$ is an LE-proximal frame. Let $a, b, c$ be elements of $L$ such that $a \delta b$ and $b \leq \bar{c}^{\delta}$. Suppose $a \bar{\delta} c$. Then, by $\left(\mathrm{pp}_{6}\right)$, there exists $h \in L$ such that $a \bar{\delta} h^{\prime}$ and $h \bar{\delta} c$.

If $b \leq h^{\prime}$, then, knowing $a \delta b$ and $\left(\mathrm{pp}_{3}\right)$, we have $a \delta h^{\prime}$ which is a contradiction. So, $b \nless h^{\prime}$, i.e., $b \wedge h \neq 0$.

If $(b \wedge h) \bar{\delta} c$, then by $\left(\mathrm{pp}_{1}\right), \bar{c}^{\delta} \leq(b \wedge h)^{\prime}$. So, by the hypothesis, $b \leq(b \wedge h)^{\prime}$. On the other hand, $b^{\prime} \leq(b \wedge h)^{\prime}$, therefore, $(b \wedge h)^{\prime}=1$ so $b \wedge h=0$, which is a contradiction. So, $(b \wedge h) \delta c$ and according to $\left(\mathrm{pp}_{5}\right), h \delta c$, which is a contradiction. Therefore, $a \delta c$, i.e., $\left(\operatorname{lp}_{6}\right)$ holds.

Example 3.13. Let $L$ be a Boolean frame and $c$ be a symmetric frame closure on $L$. Then, $\delta$ as defined in (i) is an LE-proximity and $\delta$ as defined in (ii) is an LO-proximity on $L$.

(i) $a \delta b$ iff $a \wedge b^{c} \neq 0$;

(ii) $a \delta b$ iff $a^{c} \wedge b^{c} \neq 0$. 
Proof. Let $\delta$ be defined in (i). First we show that ${ }^{-\delta}=c$. Without loss of generality, let $x \in L \backslash\{0,1\}$. Then, $x^{c} \wedge x^{c} \neq 0$ so $x^{c} \delta x$ which implies $x^{c} \leq \bar{x}^{\delta}$. According to $\left(\mathrm{fcl}_{3}\right)$ and $x^{c} \leq \bar{x}^{\delta}$, we have $x^{c} \leq\left(\bar{x}^{\delta}\right)^{c}$. Hence, the (sym) property of $c$ implies $\bar{x}^{\delta} \leq\left(x^{c}\right)^{c}$, which, by $\left(\mathrm{fcl}_{5}\right)$, implies $\bar{x}^{\delta} \leq x^{c}$. This all implies $x^{c}=\bar{x}^{\delta}$.

To $\left(\mathrm{pp}_{1}\right)$ : Let $a, b \in L$ such that $a \bar{\delta} b$, i.e., $a \wedge b^{c}=0$. So $b^{c} \leq a^{\prime}$, therefore, $\bar{b}^{\delta} \leq a^{\prime}$.

To $\left(\mathrm{pp}_{2}\right)$ : We know that $0^{c}=0$ and, for $a, b \in L, a \wedge 0=0$ and $0 \wedge b^{c}=0$, i.e., $a \bar{\delta} 0$ and $0 \bar{\delta} b$.

To $\left(\mathrm{pp}_{3}\right)$ : Let $a \delta(b \vee c)$, i.e., $a \wedge(b \vee c)^{c} \neq 0$. So, by $\left(\mathrm{fcl}_{4}\right), a \wedge\left(b^{c} \vee c^{c}\right) \neq 0$, therefore, $\left(a \wedge b^{c}\right) \vee\left(a \wedge c^{c}\right) \neq 0$. Thus, either $a \wedge b^{c} \neq 0$ or $a \wedge c^{c} \neq 0$, i.e., either $a \delta b$ or $a \delta c$. Conversely, let $a \delta b$ or $a \delta c$. Then either $a \wedge b^{c} \neq 0$ or $a \wedge c^{c} \neq 0$. In every case by $\left(\mathrm{fcl}_{2}\right), a \wedge(b \vee c)^{c} \neq 0$, i.e., $a \delta(b \vee c)$.

To $\left(\mathrm{pp}_{4}\right)$ : Let $a \in L \backslash\{0\}$. Then $a \wedge a^{c}=a(\neq 0)$, i.e., $a \delta a$.

To ( $\left.\mathrm{pp}_{5}\right)$ : Let $a \delta b$ and $a \leq c \in L$, i.e., $a \wedge b^{c} \neq 0$. Then $c \wedge b^{c} \neq 0$, i.e., $c \delta b$.

To ( $\left.\operatorname{lp}_{6}\right)$ : Let $a \delta b$ and $b \leq \bar{c}^{\delta}$, i.e., $a \wedge b^{c} \neq 0$ and $b \leq c^{c}$. Therefore, according to $\left(\mathrm{fcl}_{2}\right)$ and $\left(\mathrm{fcl}_{5}\right), b^{c} \leq c^{c}$, therefore, $a \wedge c^{c} \neq 0$, i.e., $a \delta c$.

Now let $\delta$ be defined in (ii). First we show that ${ }^{-\delta}=c$. Without loss of generality, let $x \in L \backslash\{0,1\}$. Then $\left(x^{c}\right)^{c} \wedge x^{c} \neq 0$ so $x^{c} \delta x$ which implies $x^{c} \leq \bar{x}^{\delta}$. According to $\left(\mathrm{fcl}_{3}\right)$ and $x^{c} \leq \bar{x}^{\delta}$, we have $x^{c} \leq\left(\bar{x}^{\delta}\right)^{c}$. Hence, the (sym) property of $c$ implies $\bar{x}^{\delta} \leq\left(x^{c}\right)^{c}$, which, by $\left(\mathrm{fcl}_{5}\right)$, implies $\bar{x}^{\delta} \leq x^{c}$. This all implies $x^{c}=\bar{x}^{\delta}$.

To $\left(\mathrm{pp}_{1}\right)$ : Let $a, b \in L$ such that $a \bar{\delta} b$, i.e., $a^{c} \wedge b^{c}=0$. Then $b^{c} \leq\left(a^{c}\right)^{\prime}$ and, by $\left(\mathrm{fcl}_{3}\right)$ and the complementary property, $\left(a^{c}\right)^{\prime} \leq a^{\prime}$. Therefore, $b^{c} \leq a^{\prime}$ so $\bar{b}^{\delta} \leq a^{\prime}$.

To $\left(\mathrm{pp}_{2}\right)$ : We know that $0^{c}=0$ and, for $a, b \in L, a^{c} \wedge 0=0$ and $0 \wedge b^{c}=0$, i.e., $a \bar{\delta} 0$ and $0 \bar{\delta} b$.

To $\left(\mathrm{pp}_{3}\right)$ : Let $a \delta(b \vee c)$, i.e., $a^{c} \wedge(b \vee c)^{c} \neq 0$. Then, by $\left(\mathrm{fcl}_{4}\right), a^{c} \wedge\left(b^{c} \vee c^{c}\right) \neq 0$, therefore, $\left(a^{c} \wedge b^{c}\right) \vee\left(a^{c} \wedge c^{c}\right) \neq 0$. So, either $a^{c} \wedge b^{c} \neq 0$ or $a^{c} \wedge c^{c} \neq 0$, i.e., either $a \delta b$ or $a \delta c$. Conversely, let $a \delta b$ or $a \delta c$. Then, either $a^{c} \wedge b^{c} \neq 0$ or $a^{c} \wedge c^{c} \neq 0$. In every case by $\left(\mathrm{fcl}_{2}\right), a^{c} \wedge(b \vee c)^{c} \neq 0$, i.e., $a \delta(b \vee c)$.

To $\left(\mathrm{pp}_{4}\right)$ : Let $a \in L \backslash\{0\}$. Then $a^{c} \wedge a^{c}=a^{c}(\neq 0)$, i.e., $a \delta a$.

To $\left(\mathrm{pp}_{5}\right)$ : Let $a \delta b$ and $a \leq c \in L$, i.e., $a^{c} \wedge b^{c} \neq 0$ and, by $\left(\mathrm{fcl}_{2}\right), a^{c} \leq c^{c}$. Thus $c^{c} \wedge b^{c} \neq 0$, i.e., $c \delta b$.

To $\left(\operatorname{lp}_{6}\right)$ : Let $a \delta b$ and $b \leq \bar{c}^{\delta}$, i.e., $a^{c} \wedge b^{c} \neq 0$ and $b \leq c^{c}$. Therefore, according to $\left(\mathrm{fcl}_{2}\right)$ and $\left(\mathrm{fcl}_{5}\right), b^{c} \leq c^{c}$. Hence, $a^{c} \wedge c^{c} \neq 0$ i.e. $a \delta c$.

To (sym): Let $a, b \in L$ such that $a \delta b$, i.e., $a^{c} \wedge b^{c} \neq 0$. Then $b^{c} \wedge a^{c} \neq 0$, i.e., $b \delta a$.

Definition 3.14. Let $L$ be a Boolean frame and $\Theta: L \rightarrow F F I L(L)$ satisfy the following conditions:

$\left(\operatorname{stf}_{0}\right) \quad y \notin \mathfrak{s e c} \Theta(x)$ imply $\vee\{d \in L \backslash\{0,1\} \mid y \in \mathfrak{s e c} \Theta(d)\} \leq x^{\prime} ;$

$\left(\operatorname{stf}_{1}\right) \Theta(0)=L$;

$\left(\operatorname{stf}_{2}\right) x \in L$ and $y \in \Theta(x)$ imply $x \leq y$;

$\left(\operatorname{stf}_{3}\right) x \in L$ and $z \in \Theta(x)$ imply there exists an element $t \in \Theta(x)$ such that $z \in \Theta(y)$ for each $y \in L$ with $y \leq t$.

Then, $\Theta$ is called a supertopic operator on $L$ and $(L, \Theta)$ is called a supertopic frame. 
Remark 3.15. Let $(L, \Theta)$ be an arbitrary supertopic frame. Then the following holds:

$$
x, y \in L \text { and } x \leq y \text { imply } \Theta(y) \subseteq \Theta(x) .
$$

Proof. Let $x \leq y$. We have to show that $\Theta(y) \subset \Theta(x)$. Let $z \in \Theta(y)$. By $\left(\mathrm{stf}_{3}\right)$, there exists $t \in \Theta(y)$ such that, for each $m \leq t, z \in \Theta(m)$. By $\left(\operatorname{stf}_{2}\right)$, $y \leq t$ and, by the hypothesis, $x \leq y$, so $x \leq t$. Therefore, $z \in \Theta(x)$, which implies $\Theta(y) \subseteq \Theta(x)$.

Corollary 3.16. Let $L$ be a Boolean frame and $\Theta$ be a supertopic operator on $L$. Then, we have the following relation between the supertopic operator and its corresponding PE-proximity, $\delta_{\Theta}$, on $L$.

$$
x \delta_{\Theta} y \text { iff } y \in \mathfrak{s e c} \Theta(x) .
$$

Proof. We show that $\delta_{\Theta}$ satisfies $\left(\mathrm{pp}_{1}\right)$ to $\left(\mathrm{pp}_{6}\right)$.

To $\left(\mathrm{pp}_{1}\right)$ : By $\left(\mathrm{stf}_{0}\right)$, it is obvious.

To $\left(\mathrm{pp}_{2}\right)$ : By definition of $\mathfrak{s e c}$, we know that, for every $(\emptyset \neq) A \subset L, 0$ does not belong to $\mathfrak{s e c} A$. So, $0 \notin \mathfrak{s e c} \Theta(x)$, i.e., $x \overline{\delta_{\Theta}} 0$. And also, by $\left(\operatorname{stf}_{1}\right), \Theta(0)=L$. Thus $0 \in \Theta(0)$ and, for every $y \in L$, we have $y \wedge 0=0$ so $y \notin \mathfrak{s e c} \Theta(0)$, i.e., $0 \overline{\delta_{\Theta}} y$.

To $\left(\mathrm{pp}_{3}\right)$ : Let $x \delta_{\Theta}(y \vee z)$, i.e., $y \vee z \in \mathfrak{s e c} \Theta(x)$. We know that $\Theta(x) \in F F I L(L)$, i.e., $\Theta(x)$ is a frame filter. Therefore, $\mathfrak{s e c} \Theta(x)$ is a frame grill and, by the definition of a frame grill, either $y \in \mathfrak{s e c} \Theta(x)$ or $z \in \mathfrak{s e c} \Theta(x)$, i.e., either $x \delta_{\Theta} y$ or $x \delta_{\Theta} z$. Conversely, let $x \delta_{\Theta} y$ or $x \delta_{\Theta} z$, i.e., either $y \in \mathfrak{s e c} \Theta(x)$ or $z \in \mathfrak{s e c} \Theta(x)$. In every case, $(y \vee z) \in \mathfrak{s e c} \Theta(x)$, i.e., $x \delta_{\Theta}(y \vee z)$.

To $\left(\mathrm{pp}_{4}\right)$ : Let $x \in L \backslash\{0\}$. By $\left(\operatorname{stf}_{2}\right)$, for every $z \in \Theta(x)$, we have $x \wedge z=x(\neq 0)$, therefore, $x \in \mathfrak{s e c} \Theta(x)$, i.e., $x \delta_{\Theta} x$.

To $\left(\mathrm{pp}_{5}\right)$ : Let $x \delta_{\Theta} y$ and $x \leq z$. By Remark 3.15, $x \leq z$ implies $\Theta(z) \subseteq \Theta(x)$ so, $\mathfrak{s e c} \Theta(x) \subseteq \mathfrak{s e c} \Theta(z)$. Also, from $x \delta_{\Theta} y$ we have $y \in \mathfrak{s e c} \Theta(x)$, therefore, $y \in \mathfrak{s e c} \Theta(z)$, i.e., $z \delta_{\Theta} y$.

To $\left(\mathrm{pp}_{6}\right)$ : Let $x \overline{\delta_{\Theta}} y$, i.e., $y \notin \mathfrak{s e c} \Theta(x)$. Then $\exists d \in \Theta(x)$ s.t. $y \wedge d=0$. By $\left(\operatorname{stf}_{3}\right)$, there exists $z \in \Theta(x)$ such that $d \in \Theta(t)$ for each $t \in L$ with $t \leq z$. Since $z \in \Theta(x), z^{\prime} \notin \mathfrak{s e c} \Theta(x)$, i.e., $x \overline{\delta_{\Theta}} z^{\prime}$. Since, for each $t \in L$ with $t \leq z$, we have $d \in \Theta(t)$ and $y \wedge d=0$, we have $y \notin \mathfrak{s e c} \Theta(t)$, i.e., $t \overline{\delta_{\Theta}} y$.

Definition 3.17. Let $L$ be a Boolean frame and $\xi$ be a subset of $P L$ which satisfies the following conditions:

$\left(\right.$ nf $\left._{1}\right)$ If $A<<B$ and $B \in \xi$, then $A \in \xi$;

$\left(\mathrm{nf}_{2}\right)$ If $\bigwedge A \neq 0$, then $A \in \xi$;

$\left(\mathrm{nf}_{3}\right) \emptyset \neq \xi \neq P L$

(nf $\left._{4}\right)$ If $(A \bigvee B) \in \xi$, then $A \in \xi$ or $B \in \xi$;

$\left(\mathrm{nf}_{5}\right) A \subset L \backslash\{0\}$ and $\left\{\bar{x}^{\xi}: x \in A\right\} \in \xi$ imply $A \in \xi$, where $\overline{0}^{\xi}:=0, \overline{1}^{\xi}:=1$ and $\bar{x}^{\xi}:=\vee\{z \in L \backslash\{0,1\}:\{x, z\} \in \xi\}$. The map ${ }^{-\xi}: L \rightarrow L$ is called a $\xi$-closure.

Then $\xi$ is called a nearness on $L$, and the pair $(L, \xi)$ is called a nearness frame. Let $(L, \xi)$ and $(M, \eta)$ be two nearness frames. A frame homomorphism $f: L \rightarrow M$ is called a near frame (or shortly,nf-map) if and only if it satisfies the following condition: 
(nf) $A \in \xi$ implies $f(A) \in \eta$.

Lemma 3.18. For every nearness frame $(L, \xi)$, the $\xi$-closure is a symmetrical frame-closure.

Proof. We show that $\xi$-closure satisfies $\left(\mathrm{fcl}_{1}\right)$ to $\left(\mathrm{fcl}_{5}\right)$ and (sym).

To $\left(\mathrm{fcl}_{1}\right)$ : By definition, it is obvious.

To $\left(\mathrm{fcl}_{2}\right)$ : Let $x, z \in L$ such that $x \leq z$. Without loss of generality, let $x, z \in$ $L \backslash\{0,1\}$. Then we have to verify $\bar{x}^{\xi} \leq \bar{z}^{\xi}$. Now, for $y \in L \backslash\{0,1\}$ with $\{x, y\} \in \xi$, by the assumption, $\{y, z\}<<\{y, x\}$, and, according to $\left(\operatorname{nf}_{1}\right)$, we have $\{y, z\} \in \xi$. Consequently, $y \leq \bar{z}^{\xi}$, because $\bar{z}^{\xi}$ is the join of a set. Hence, $\bar{x}^{\xi} \leq \bar{z}^{\xi}$ because $\bar{x}^{\xi}$ is the join of a set.

To $\left(\mathrm{fcl}_{3}\right)$ : By definition, it is obvious.

To (fcl $\mathrm{f}_{4}$ ): Without loss of generality, let $x, z \in L \backslash\{0,1\}$. Now, for $y \in L \backslash\{0,1\}$ with $\{y,(x \vee z)\} \in \xi$, according to $\left(\mathrm{nf}_{1}\right)$ and $\left(\mathrm{nf}_{4}\right)$ we have $\{x, y\} \in \xi$ or $\{y, z\} \in \xi$. In the first case, we have $y \leq \bar{x}^{\xi}$ and, in the second case, we have, $y \leq \bar{z}^{\xi}$. Therefore, in every case, $y \leq \bar{x}^{\xi} \vee \bar{z}^{\xi}$. Hence, $\overline{x \vee} z^{\xi} \leq \bar{x}^{\xi} \vee \bar{z}^{\xi}$ because $\overline{x \vee} \bar{z}^{\xi}$ is the join of a set.

To $\left(\mathrm{fcl}_{5}\right)$ : Without loss of generality, let $x \in L \backslash\{0,1\}$. Now, for $y \in L \backslash\{0,1\}$ with $\left\{\bar{x}^{\xi}, y\right\} \in \xi$, we have $\left\{\bar{x}^{\xi}, \bar{y}^{\xi}\right\}<<\left\{\bar{x}^{\xi}, y\right\}$ and, by $\left(\mathrm{nf}_{1}\right)$, we have $\left\{\bar{x}^{\xi}, \bar{y}^{\xi}\right\} \in \xi$ and, according to $\left(\mathrm{nf}_{5}\right),\{x, y\} \in \xi$. Consequently, $y \leq \bar{x}^{\xi}$. Hence, $\overline{\left(\bar{x}^{\xi}\right)} \bar{x}^{\xi} \leq \bar{x}^{\xi}$ because $\overline{\left(\bar{x}^{\xi}\right)}$ is the join of a set.

To (sym): Without loss of generality, let $x, z \in L \backslash\{0,1\}$ such that $x \leq \bar{z}^{\xi}$. By $\left(\mathrm{nf}_{2}\right)$ we have $\left\{x, \bar{z}^{\xi}\right\} \in \xi$ and since $\left\{\bar{x}^{\xi}, \bar{z}^{\xi}\right\}<<\left\{x, \bar{z}^{\xi}\right\}$ by $\left(\mathrm{nf}_{1}\right)$, we have $\left\{\bar{x}^{\xi}, \bar{z}^{\xi}\right\} \in \xi$. Therefore, by $\left(\mathrm{nf}_{5}\right),\{x, z\} \in \xi$, hence $z \leq \bar{x}^{\xi}$ because $\bar{x}$ is the join of a set.

Definition 3.19. Let $L$ be a Boolean frame. A function $N: L \rightarrow P(P(L))$ is called a supernear frame operator (snf-operator), and the pair $(L, N)$ is called a supernear frame, if and only if $N$ satisfies the following conditions:

$\left(\operatorname{snf}_{1}\right) x \in L, A, D \subset L$ and $A<<D \in N(x)$ imply $A \in N(x)$;

$\left(\operatorname{snf}_{2}\right) \quad x \in L$ implies $N(x) \neq \emptyset$;

$\left(\operatorname{snf}_{3}\right) \quad A \in N(0)$ implies $A=\emptyset$ and $\{0\} \notin N(1)$;

$\left(\operatorname{snf}_{4}\right) \quad(0 \neq) x \in L$ implies $\{x\} \in N(x)$;

$\left(\operatorname{snf}_{5}\right) x, y \in L$ and $x \leq y$ imply $N(x) \subseteq N(y)$;

$\left(\operatorname{snf}_{6}\right) x \in L$ and $A, D \subset L$ and $A \vee D \in N(x)$ imply $A \in N(x)$ or $D \in N(x)$;

$\left(\right.$ snf $\left._{7}\right) x \in L, A \subset L$ and $\left\{\bar{z}^{N}: z \in A\right\} \in N(x)$ imply $A \in N(x)$, where $\overline{0}^{N}:=0$, $\overline{1}^{N}:=1$ and for $z \in L \backslash\{0,1\}, \bar{z}^{N}:=\bigvee\{x \in L \backslash\{0,1\}:\{z\} \in N(x)\}$. The map $^{-N}: L \rightarrow L$ is called an $N$-closure on $L$.

For $x \in L$ and $A \in N(x), A$ is called a $x$-near set in $N$. For supernear frames $\left(L_{1}, N_{1}\right)$ and $\left(L_{2}, N_{2}\right)$, a frame homomorphism $g: L_{1} \rightarrow L_{2}$ is called a supernear frame map (or shortly, snf-map) if and only if it satisfies the following condition:

(snf) $x \in L_{1}$ and $A \in N_{1}(x)$ imply $g[A] \in N_{2}(g(x))$.

The corresponding category is denoted by SNFRM. 
Example 3.20. Let $L$ be a Boolean frame. Then, the following functions are supernear frame operators on $L$.

$$
\begin{aligned}
& N_{1}(x):= \begin{cases}\{\emptyset\} & \text { if } x=0, \\
\{A \subset L \mid 0 \notin A\} & \text { otherwise, }\end{cases} \\
& N_{2}(x):= \begin{cases}\{\emptyset\} & \text { if } x=0, \\
\left\{A \subset L \mid x \in \mathfrak{s e c}\left\{\bar{a}^{c} \mid a \in A\right\}\right. & \text { otherwise, }\end{cases}
\end{aligned}
$$

where $c$ is the symmetrical frame closure on $L$.

Proof. Easily, we can see that $N_{1}$ is a supernear frame operator on $L$. Now we show that $N_{2}$ is also a supernear frame operator on $L$.

To $\left(\operatorname{snf}_{1}\right)$ : Let $A<<D$ and $D \in N_{2}(x)$. Then, for every $d \in D, x \wedge \bar{d}^{c} \neq 0$. Also, for every $a \in A$, there exists $d \in D$ s.t. $d \leq a$. Therefore, by $\left(\mathrm{fcl}_{2}\right)$, we have $\bar{d}^{c} \leq \bar{a}^{a}$ and this all implies that for every $a \in A, x \wedge \bar{a}^{c} \neq 0$. Hence, $A \in N_{2}(x)$.

To (snf2): By definition, it is obvious.

To (snf3): By definition, it is obvious. To (snf4): Let $(0 \neq) x \in L$. Then, by $\left(\mathrm{fcl}_{3}\right), x^{c} \wedge x=x(\neq 0)$, so $\{x\} \in N_{2}(x)$.

To (snf5): Let $x \leq y$ and $A \in N_{2}(x)$. For every $a \in A$, we have $x \wedge \bar{a}^{c} \neq 0$. Therefore, for every $a \in A$, we have $y \wedge \bar{a}^{c} \neq 0$, i.e., $A \in N_{2}(y)$.

To (snf6): Let $A \vee D \in N_{2}(x)$. For every $a \in A$ and for every $d \in D$, we have, $x \wedge \overline{(a \vee d)}^{c} \neq 0$. By $\left(\mathrm{fcl}_{4}\right), x \wedge\left(\bar{a}^{c} \vee \bar{d}^{c}\right) \neq 0$, so, for every $a \in A$ and for every $d \in D$, either $x \wedge \bar{a}^{c} \neq 0$ or $x \wedge \bar{d}^{c} \neq 0$. Now suppose for $a_{1} \in A, x \wedge{\overline{a_{1}}}^{c}=0$ and, for $d_{1} \in D, x \wedge{\overline{d_{1}}}^{c}=0$. Then $x \wedge\left({\overline{a_{1}}}^{c} \vee{\overline{d_{1}}}^{c}\right)=0$, which is a contradiction. So, either, for all $a \in A, x \wedge \bar{a}^{c} \neq 0$ or, for all $d \in D, x \wedge \bar{d}^{c} \neq 0$, i.e., either $A \in N_{2}(x)$ or $D \in N_{2}(x)$.

To $\left(\operatorname{snf}_{7}\right)$ : First we show that $-N_{2}={ }^{-c}$. Let $z \in L$. If $z \in\{0,1\}$, then obviously $\bar{z}^{c}=\bar{z}^{N_{2}}$. So, let $z \in L \backslash\{0,1\}$. Then $\bar{z}^{N_{2}}=\vee\{x \in L \backslash\{0,1\} \mid\{z\} \in$ $\left.N_{2}(x)\right\}=\vee\left\{x \in L \backslash\{0,1\} \mid x \wedge \bar{z}^{c} \neq 0\right\}$, therefore, $\bar{z}^{c} \leq \bar{z}^{N_{2}}$. Now we have $\bar{z}^{c} \leq \bar{z}^{N_{2}}$ and, by $\left(\mathrm{fcl}_{3}\right), \bar{z}^{c} \leq{\overline{\left(\bar{z}^{N_{2}}\right)}}^{c}$. Since ${ }^{-c}$ is symmetrical, we have $\bar{z}^{N_{2}} \leq{\overline{\left(\bar{z}^{c}\right)}}^{c}$ and, by $\left(\mathrm{fcl}_{5}\right), \bar{z}^{N_{2}} \leq \bar{z}^{c}$. Therefore, this all implies $\bar{z}^{N_{2}}=\bar{z}^{c}$. Now let $\left\{\bar{a}^{N_{2}} \mid a \in\right.$ $A\} \in N(x)$. By definition, $x \in \mathfrak{s e c}\left\{{\overline{\left(\bar{a}^{N_{2}}\right)}}^{c} \mid a \in A\right\}$ and, since ${ }^{-N_{2}}={ }^{-c}$, we have $x \in \mathfrak{s e c}\left\{\overline{\left(\bar{a}^{c}\right)} \mid a \in A\right\}$. By $\left(\mathrm{fcl}_{3}\right)$ and $\left(\mathrm{fcl}_{5}\right), x \in \mathfrak{s e c}\left\{\bar{a}^{c} \mid a \in A\right\}$ and, by definition, it means $A \in N_{2}(x)$.

Example 3.21. Let $L$ be a Boolean frame. Then the following functions are supernear frame operators on $L$.

(i) Let $c$ be a symmetric frame closure on $L$. Then we define the corresponding functions from $L$ to $P(P(L))$ by setting:

$$
N^{c}(x):= \begin{cases}\{\emptyset\} & \text { if } x=0, \\ \left\{A \subset L \backslash\{0\} \mid x^{c} \in \mathfrak{s e c}\left\{a^{c} \mid a \in A\right\}\right\} & \text { otherwise. }\end{cases}
$$

(ii) Let $(L, \xi)$ be a nearness frame. Then we define the corresponding function from $L$ to $P(P(L))$ by setting:

$$
N_{\xi}(x):= \begin{cases}\{\emptyset\} & \text { if } x=0, \\ \{A \subset L \mid\{x\} \cup A \in \xi\} & \text { otherwise. }\end{cases}
$$


(iii) Let $(L, \delta)$ be a PE-proximal frame and $\left(L, \Theta_{\delta}\right)$ be its corresponding supertopic frame. Then we define the corresponding function from $L$ to $P(P(L))$ by setting:

$$
N_{\delta}(0):= \begin{cases}\{\emptyset\} & \text { if } x=0, \\ \{A \subset L \backslash\{0\} \mid A \subset \delta(x)\} & \text { otherwise, }\end{cases}
$$

where $\delta(x):=\{z \in L \mid x \delta z\}$.

Definition 3.22. A supernear frame $(L, N)$ is called a paranear frame if and only if $N$ has the following symmetric property:

(sym) $x \in L \backslash\{0\}$ and $A \in N(x)$ imply $\{x\} \cup A \in \bigcap\{N(z) \mid z \in A \cup\{x\}\}$.

The corresponding category is denoted by PNFRM which is full subcategory of SNFRM.

Definition 3.23. A supernear frame $(L, N)$ is called a supergrill frame if and only if it satisfies the following condition:

(gri) $x \in L \backslash\{0\}$ and $A \in N(x)$ imply $\exists G \in F G R L(L) \cap N(x)$ such that $A \subset G$.

Definition 3.24. A supernear frame $(L, N)$ is called conic if and only if it satisfies the following condition:

(cnc) $x \in L$ implies $\cup N(x) \in N(x)$, where

$$
\cup N(x):=\{z \in L \mid \exists A \in N(x) \text { such that } z \in A\} .
$$

Theorem 3.25. The category LOProxFrm, whose objects are LO-proximity frame, is isomorphic to a full subcategory of SNFRM.

Proof. By considering Example 3.21 (iii), we can easily see that the statement holds.

Theorem 3.26. Supertopic frames are in one to one correspondence to specific conic supergrill frames.

Proof. Let $(L, \Theta)$ be a given supertopic frame. Then, we define $N_{\Theta}$ on $L$ by setting, $N_{\Theta}(x):=\{A \subset L \backslash\{0\} \mid A \subset \mathfrak{s e c} \Theta(x)\}$. Now we show that $N_{\Theta}$ is a conic supergrill frame.

To $\left(\operatorname{snf}_{1}\right)$ : Let $A<<D$ and $D \in N_{\Theta}(x)$, i.e., $D \subset \mathfrak{s e c} \Theta(x)$. Thus, for every $d \in D$ and every $y \in \Theta(x)$, we have $d \wedge y \neq 0$. On the other hand, for every $a \in A$, there exists $d \in D$ such that $d \leq a$. Thus, for every $a \in A$ and every $y \in \Theta(x)$, we have $a \wedge y \neq 0$, i.e., $A \subset \mathfrak{s e c} \Theta(x)$, so $A \in N_{\Theta}(x)$.

To $\left(\operatorname{snf}_{2}\right)$ : By definition, it is obvious.

To $\left(\operatorname{snf}_{3}\right)$ : By definition, it is obvious.

To $\left(\operatorname{snf}_{4}\right)$ : Let $(0 \neq) x \in L$. By $\left(\operatorname{stf}_{2}\right)$, we know that, for every $y \in \Theta(x), x \leq y$. So, $x \wedge y=x(\neq 0)$ and, therefore, $x \in \mathfrak{s e c} \Theta(x)$.

To $\left(\operatorname{snf}_{5}\right)$ : Let $x \leq y$. By Remark 3.15, $\Theta(y) \subset \Theta(x)$, therefore, $\mathfrak{s e c} \Theta(x) \subset$ $\mathfrak{s e c} \Theta(y)$. Now let $A \in N_{\Theta}(x)$. Then $A \subset \mathfrak{s e c} \Theta(x)$ and, therefore, $A \subset \mathfrak{s e c} \Theta(y)$, i.e., $A \in N_{\Theta}(y)$.

To $\left(\operatorname{snf}_{6}\right)$ : Let $A \vee D \in N_{\Theta}(x)$, i.e., $A \vee D \subset \mathfrak{s e c} \Theta(x)$. Then, for every $y \in \Theta(x)$, $(a \vee d) \wedge y \neq 0$ when $a \in A$ and $d \in D$. Therefore, for every $y \in \Theta(x)$, either $a \wedge y \neq 0$ or $d \wedge y \neq 0$. If for $a_{1} \in A, a_{1} \wedge y=0$ and for $d_{1} \in D, d_{1} \wedge y=0$, then 
$y \wedge\left(a_{1} \vee d_{1}\right)=0$, which is a contradiction. So, either, for all $a \in A, a \wedge y \neq 0$ or, for all $d \in D, d \wedge y \neq 0$, i.e., either $A \in N_{\Theta}(x)$ or $D \in N_{\Theta}(x)$.

To $\left(\operatorname{snf}_{7}\right)$ : Let $\left\{\bar{a}^{N_{\Theta}} \mid a \in A\right\} \in N_{\Theta}(x)$ so $\left\{\bar{a}^{N_{\Theta}} \mid a \in A\right\} \subset \mathfrak{s e c} \Theta(x)$. Now suppose $A \notin N_{\Theta}(x)$. Then there exists $a \in A$ such that $a \notin \mathfrak{s e c} \Theta(x)$ which means there exists $k \in \Theta(x)$ such that $a \wedge k=0$. Therefore, by $\left(\operatorname{stf}_{3}\right)$, there exists $h \in \Theta(x)$ such that, for every $s \leq h, k \in \Theta(s)$. Since $h \in \Theta(x)$, we have $h^{\prime} \notin \mathfrak{s e c} \Theta(x)$. Also, we know that $k \in \Theta(h)$ and $a \wedge k=0$, hence $a \notin \mathfrak{s e c} \Theta(h)$. Therefore, we have two possibilities.

(1) If $\bar{a}^{N_{\Theta}} \leq h^{\prime}$, then $\left\{h^{\prime}\right\}<<\left\{\bar{a}^{N_{\Theta}}\right\}$ and, by knowing $\left\{\bar{a}^{N_{\Theta}}\right\} \in N_{\Theta}(x)$ and using $\left(\operatorname{snf}_{1}\right.$ ), we have $\left\{h^{\prime}\right\} \in N_{\Theta}(x)$, i.e., $h^{\prime} \in \mathfrak{s e c} \Theta(x)$, which is a contradiction.

(2) If $\bar{a}^{N} \not h^{\prime}$, then $\bar{a}^{N} \wedge h \neq 0$. Therefore, we have again two possibilities.

(i) If $\{a\} \notin N_{\Theta}\left(\bar{a}^{N} \wedge h\right)$, then $a \notin \mathfrak{s e c} \Theta\left(\bar{a}^{N} \wedge h\right)$. So, by $\left(\operatorname{stf}_{0}\right)$ and the definition of N-closure, we have $\bar{a}^{N} \leq\left(\bar{a}^{N} \wedge h\right)^{\prime}$. Since $\left(\bar{a}^{N}\right)^{\prime} \leq\left(\bar{a}^{N} \wedge h\right)^{\prime},\left(\bar{a}^{N} \wedge h\right)^{\prime}=1$, so $\left(\bar{a}^{N} \wedge h\right)=0$, which is a contradiction.

(ii) If $\{a\} \in N_{\Theta}\left(\bar{a}^{N} \wedge h\right)$, then, by $\left(\operatorname{snf}_{5}\right)$, we have $\{a\} \in N(h)$. Therefore, $a \in \mathfrak{s e c} \Theta(h)$, which is a contradiction.

So, $A \subset \mathfrak{s e c} \Theta(x)$, i.e., $A \in N_{\Theta}(x)$. Therefore, $\left(L, N_{\Theta}\right)$ is a supernear frame. Also, it is obvious that $\left(L, N_{\Theta}\right)$ is conic.

Now we show that $\left(L, N_{\Theta}\right)$ is a supergrill frame. For every $A \in N_{\Theta}(x)$, we know that $A \subset \mathfrak{s e c} \Theta(x)$ and, by Proposition 3.3, sec $\Theta(x) \in F G R L(L)$. This implies $\left(L, N_{\Theta}\right)$ is a supergrill frame.

Conversely, let there be given specific conic supergrill frame $(L, N)$. Ten we define $\Theta_{N}$ on $L$ by setting, $\Theta_{N}(x):=\mathfrak{s e c}(\cup N(x))$.

Remark 3.27. The category STFRM, whose objects are supertopic frames, is isomorphic to a full subcategory of SNFRM.

Proof. By Theorem 3.26, it is easy to see that it holds.

Theorem 3.28. The category NFRM is isomorphic to a full subcategory of SNFRM.

Proof. Considering Example 3.21 (ii), it is easy to see that it holds.

Lemma 3.29. For every supernear frame $(L, N)$, the $N$-closure is a frameclosure.

Proof. We show that $N$-closure satisfies $\left(\mathrm{fcl}_{1}\right)$ to $\left(\mathrm{fcl}_{5}\right)$.

To $\left(\mathrm{fcl}_{1}\right)$ : By definition, it is obvious.

To $\left(\mathrm{fcl}_{2}\right)$ : Let $x, z \in L$ such that $x \leq z$. Without loss of generality, let $x, z \in$ $L \backslash\{0,1\}$. We have to verify $\bar{x}^{N} \leq \bar{z}^{N}$. By the assumption, we know that $\{z\}<<$ $\{x\}$, so, for arbitrary $y \in L \backslash\{0,1\}$ with $\{x\} \in N(y)$, by $\left(\operatorname{snf}_{1}\right)$, we have $\{z\} \in N(y)$. Consequently, $y \leq \bar{z}^{N}$ because $\bar{z}^{N}$ is the join of a set. Hence, $\bar{x}^{N} \leq \bar{z}^{N}$ because $\bar{x}^{N}$ is the join of a set.

To $\left(\mathrm{fcl}_{3}\right)$ : By definition, it is obvious.

To $\left(\mathrm{fcl}_{4}\right)$ : Without loss of generality, let $x, z \in L \backslash\{0,1\}$. For arbitrary $y \in$ $L \backslash\{0,1\}$ with $\{x \vee z\} \in N(y)$, by $\left(\operatorname{snf}_{6}\right)$, we have $\{x\} \in N(y)$ or $\{z\} \in N(y)$. In the first case we have $y \leq \bar{x}^{N}$, and in the second case we have, $y \leq \bar{z}^{N}$. Therefore, in every case, $y \leq \bar{x}^{N} \vee \bar{z}^{N}$. Hence, $\overline{x \vee z^{N}} \leq \bar{x}^{N} \vee \bar{z}^{N}$ because $\overline{x \vee}^{N}$ is the join of a set. 
To $\left(\mathrm{fcl}_{5}\right)$ : Without loss of generality, let $x \in L \backslash\{0,1\}$. For arbitrary $y \in$ $L \backslash\{0,1\}$ with $\left\{\bar{x}^{N}\right\} \in N(y)$, by $\left(\operatorname{snf}_{7}\right)$, we have $\{x\} \in N(y)$ and, consequently, $y \leq \bar{x}^{N}$. Hence, ${\overline{\left(\bar{x}^{N}\right)}}^{N} \leq \bar{x}^{N}$ because ${\overline{\left(\bar{x}^{N}\right)}}^{N}$ is the join of a set.

Definition 3.30. Let $L, M$ be Boolean frames with frame closures $c_{L}$ and $c_{M}$ respectively. A frame homomorphism $g: L \rightarrow M$ is called frame-continuous $(f c-m a p)$ if and only if it satisfies the following condition:

(fc) $x \in L$ implies $g\left(x^{c_{L}}\right) \leq(g(x))^{c_{M}}$.

Lemma 3.31. Let $\left(L_{1}, N_{1}\right)$ and $\left(L_{2}, N_{2}\right)$ be supernear frames and $g: L_{1} \rightarrow L_{2}$ be an snf-map. Then, $g:\left(L_{1},-N_{1}\right) \rightarrow\left(L_{2},-N_{2}\right)$ is frame-continuous.

Proof. Without loss of generality, for $x \in L_{1} \backslash\{0,1\}$ we have to show that $g\left(\bar{x}^{N_{1}}\right) \leq \overline{g(x)}^{N_{2}}$. It is sufficient to prove that $\{g(x)\} \in N_{2}\left(g\left(\bar{x}^{N_{1}}\right)\right)$. By the assumption, according to $\left(\operatorname{snf}_{4}\right)$, we know that $\{x\} \in N_{1}(x)$ and, since $g$ is snfmap, $\{g(x)\} \in N_{2}(g(x))$. Since $g$ is a frame homomorphism and preserves arbitrary joins, we have $g(x) \leq g\left(\bar{x}^{N_{1}}\right)$. Then, by $\left(\operatorname{snf}_{5}\right)$ we get $N_{2}(g(x)) \subset N_{2}\left(g\left(\bar{x}^{N_{1}}\right)\right)$. Therefore, $\{g(x)\} \in N_{2}\left(g\left(\bar{x}^{N_{1}}\right)\right)$.

Definition 3.32. A supernear frame $(L, N)$ is called connected if and only if it satisfies the following condition:

(cnc) $x, z \in L$ implies $N(x \vee z) \subset N(x) \cup N(z)$.

Definition 3.33. A supernear frame $(L, N)$ is called dense if and only if it satisfies the following condition:

(d) $x \in L$ implies $N\left(\bar{x}^{N}\right) \subset N(x)$.

Lemma 3.34. Every paranear frame is connected and dense.

Proof. Let $(L, N)$ be an arbitrary paranear frame. We show that it is connected and dense.

To (cnc): Without loss of generality, for $x, z \in L \backslash\{0,1\}$, let $A \in N(x \vee z)$. Then, by the symmetric property of $N$, we have $\{x \vee z\} \cup A \in N(x \vee z)$. Hence, by $\left(\operatorname{snf}_{1}\right)$, $(\{x\} \cup A) \vee(\{z\} \cup A) \in N(x \vee z)$ and, according to $\left(\operatorname{snf}_{6}\right),\{x\} \cup A \in N(x \vee z)$ or $\{z\} \cup A \in N(x \vee z)$. In the first case, according to the symmetric property of $N$, we have $\{x \vee z\} \cup(\{x\} \cup A) \in N(x)$ and, by $\left(\operatorname{snf}_{1}\right), A \in N(x)$. The second case can be verified analogously. Hence, in every case we have $A \in N(x) \cup N(z)$.

To (d): Let $A \in N\left(\bar{x}^{N}\right)$. Then, by the symmetric property of $N$, we have $\left\{\bar{x}^{N}\right\} \cup A \in N\left(\bar{x}^{N}\right)$. Then, according to $\left(\operatorname{snf}_{1}\right),\left\{\bar{x}^{N}\right\} \cup\left\{\bar{a}^{N}: a \in A\right\} \in N\left(\bar{x}^{N}\right)$. Consequently, by $\left(\operatorname{snf}_{7}\right),\{x\} \cup A \in N\left(\bar{x}^{N}\right)$ and, with respect to the symmetric property of $N$, we have $\left\{\bar{x}^{N}\right\} \cup(\{x\} \cup A) \in N(x)$. Therefore, by $\left(\operatorname{snf}_{1}\right)$, we have $A \in N(x)$.

Corollary 3.35. For a paranear frame $(L, N)$, the $N$-closure on $L$ is a symmetrical frame closure.

Proof. We show that $N$-closure satisfies (sym). Let $x, z \in L \backslash\{0,1\}$ and $x \leq \bar{z}^{N}$. By $\left(\operatorname{snf}_{4}\right)$, we have $\{x\} \in N(x)$. Therefore, by the assumption and $\left(\operatorname{snf}_{5}\right)$, we have $\{x\} \in N\left(\bar{z}^{N}\right)$. Also, by Lemma 3.34, we know that $N$ is dense. So, $\{x\} \in N(z)$, hence $z \leq \bar{x}^{N}$ because $\bar{x}^{N}$ is the join of a set. 


\section{TOPOlOGiCAl FRAME EXTENSIONS AND RELATED PARANEAR FRAMES}

Topological extensions [1] play an important role in the theory of proximities and nearness. For example, the Smirnov compactification [12] of a proximity space $X$ is a compact Hausdorff space $Y$ which contains $X$ as a dense subspace and for which it is true that a pair of subsets of $X$ is near if and only if their closures in $Y$ meet. M. W. Lodato generalized this result to weaker conditions for the proximity and the space $Y$ using bunches for the characterization of the extension. V. M. Ivanova and A. Ivanov [6] studied contiguity spaces and bicompact extensions. H. Herrlich [4] found a useful generalization of contiguity spaces by introducing nearness spaces, and H. L. Bentley [2] showed that those nearness spaces which can be extended to topological ones have a neat internal characterization. D. Doitchinov [3] introduced the notion of supertopological spaces in order to construct a unified theory of topological, proximity and uniform spaces, and he proved a certain relationship of some special classes of supertopologies (called b-supertopologies) to compactly determined extensions. Then, D. Leseberg [9] introduced supernearness spaces and the corresponding topological extensions. Now we will give a corresponding description in the realm of Boolean frames.

Definition 4.1. A topological frame extension consists of a triple $(e, L, Y)$, where $L$ is a Boolean frame with frame-closure $c: L \rightarrow L, Y:=\left(X \cup\{\infty\}, c l_{Y}\right)$ is a topological space with the underlying set $X$ and the corresponding closure operator $c l_{Y}$, and $e: L \rightarrow Y$ is a function which satisfies the following conditions: $\left(\mathrm{tfe}_{0}\right) \quad e(0)=\infty$ while $z \in L \backslash\{0\}$ implies $e(z) \neq \infty$;

$\left(\mathrm{tfe}_{1}\right) \operatorname{cl}_{Y}(e[L \backslash\{0\}])=X$ (which means that the image of $L \backslash\{0\}$ under $e$ is dense in $X)$;

$\left(\mathrm{tfe}_{2}\right) z \in L \backslash\{0,1\}$ implies $z^{c}=\vee e^{-1}\left[c l_{Y}(\{e(z)\})\right]$;

$\left(\mathrm{tfe}_{3}\right) z \in L$ implies $e\left(z^{c}\right) \in c l_{Y}(\{e(z)\})$;

$\left(\mathrm{tfe}_{4}\right) z_{1}, z_{2} \in L$ and $(0 \neq) z_{1} \leq z_{2}$ imply $c l_{Y}\left(\left\{e\left(z_{1}\right)\right\}\right) \subset c l_{Y}\left(\left\{e\left(z_{2}\right)\right\}\right)$;

$\left(\mathrm{tfe}_{5}\right) \forall z_{1}, z_{2} \in L, c l_{Y}\left(\left\{e\left(z_{1} \vee z_{2}\right)\right\}\right) \subset c l_{Y}\left(\left\{e\left(z_{1}\right)\right\}\right) \cup c l_{Y}\left(\left\{e\left(z_{2}\right)\right\}\right)$.

For topological frame extensions $(e, L, Y)$ and $\left(e^{\prime}, L^{\prime}, Y^{\prime}\right)$, a pair $(g, h)$ of maps $g: L \rightarrow L^{\prime}$ and $h: Y \rightarrow Y^{\prime}$ is called a tfe-morphism if and only if $g$ is a frame continuous map and $h$ is a continuous map such that the following diagram commutes:

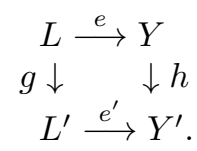

If $(g, h):(e, L, Y) \rightarrow\left(e^{\prime}, L^{\prime}, Y^{\prime}\right)$ and $\left(g^{\prime}, h^{\prime}\right):\left(e^{\prime}, L^{\prime}, Y^{\prime}\right) \rightarrow\left(e^{\prime \prime}, L^{\prime \prime}, Y^{\prime \prime}\right)$ are tfe-morphisms, then they can be composed by the following rule: $\left(g^{\prime}, h^{\prime}\right) \circ(g, h):=$ $\left(g^{\prime} \circ g, h^{\prime} \circ h\right):(e, L, Y) \rightarrow\left(e^{\prime \prime}, L^{\prime \prime}, Y^{\prime \prime}\right)$, where $\circ$ denotes the composition of maps. The corresponding category is denoted by TFE.

Definition 4.2. A topological frame extension $(e, L, Y)$ is called

(i) Strict topological frame extension if and only if it satisfies the following condition: 
(str) $\left\{c l_{Y}(e[A]): A \subset L \backslash\{0\}\right\}$ forms a base for the closed subsets of $X$. The corresponding category is denoted by STRTFE - it is a full subcategory of TFE.

(ii) Symmetric topological frame extension if and only if in addition it satisfies the following condition:

(sym) $x \in L \backslash\{0\}$ and $y \in \operatorname{cl}_{Y}(\{e(x)\})$ imply $e(x) \in c_{Y}(\{y\})$.

The corresponding category is denoted by SYMTFE - it is a full subcategory of TFE.

Example 4.3. Let $(e, L, Y)$ be a topological frame extension. Then, the following function is a supernear operator on $L$ :

$$
N_{e}(x):= \begin{cases}\{\emptyset\} & \text { if } x=0, \\ \left\{A \subset L \backslash\{0\} \mid c l_{Y}(\{e(x)\}) \in \mathfrak{s e c}\left\{c l_{Y}(\{e(a)\}) \mid a \in A\right\}\right\} & \text { otherwise. }\end{cases}
$$

Lemma 4.4. Let $(e, L, Y)$ be a symmetric topological frame extension. Then $\left(L, N_{e}^{S}\right)$ is a paranear frame such that, for each $x \in L$, we have $x^{c}=\bar{x}^{N_{e}^{S}}$ where

$$
N_{e}^{S}(x):= \begin{cases}\{\emptyset\} & \text { if } x=0, \\ \left\{A \subset L \backslash\{0\} \mid \bigcap\left\{c l_{Y}(\{e(z)\}) \mid z \in A \cup\{x\}\right\} \neq \emptyset\right\} & \text { otherwise. }\end{cases}
$$

Proof. First we show the equality of the frame-closures $c$ and $-N_{e}^{S}$. Without loss of generality, let $x \in L \backslash\{0,1\}$. Considering that $c l_{Y}$ is a topological closure operator and by $\left(\mathrm{tfe}_{3}\right)$, we have $e\left(x^{c}\right) \in c l_{Y}(\{e(x)\}) \cap c l_{Y}\left(\left\{e\left(x^{c}\right)\right\}\right)$, so $\{x\} \in$ $N_{e}^{S}\left(x^{c}\right)$. Therefore, $x^{c} \leq \bar{x}^{N_{e}^{S}}$. Conversely, we show that $x^{c}$ is an upper bound of the set $A:=\left\{z \in L \backslash\{0,1\} \mid\{x\} \in N_{e}^{S}(z)\right\}$. Let $z \in A$. Then $\{x\} \in N_{e}^{S}(z)$, hence $c l_{Y}(\{e(x)\}) \cap c l_{Y}(\{e(z)\}) \neq \emptyset$. So, $\exists y \in c l_{Y}(\{e(z)\})$ and $y \in c l_{Y}(\{e(x)\})$. Since $c l_{Y}$ is topological closure operator and by (sym), we have $e(z) \in c l_{Y}(\{y\}) \subset$ $c l_{Y}(\{e(x)\})$. Therefore, $z \in e^{-1}\left[c l_{Y}(\{e(x)\})\right]$ and, by $\left(\mathrm{tfe}_{2}\right)$, we get $z \leq x^{c}$ so $\bar{x}^{N_{e}^{S}} \leq x^{c}$. Therefore, $x^{c}=\bar{x}^{N_{e}^{S}}$.

Secondly we have to verify that $N_{e}^{S}$ satisfies the axioms $\left(\operatorname{snf}_{1}\right)$ to $\left(\operatorname{snf}_{7}\right)$ and (sym).

To $\left(\operatorname{snf}_{1}\right)$ : Let $x \in L$ and $A, D \subset L$. Without loss of generality, let $x \neq 0$ and $A<<D \in N_{e}^{S}(x)$. Then $\bigcap\left\{c l_{Y}(\{e(d)\}): d \in D \cup\{x\}\right\} \neq \emptyset$ and $0 \notin A$. Now, let $a$ be an arbitrary element of $A$. Then there exists $d \in D$ such that $d \leq a$. Consequently, by $\left(\mathrm{tfe}_{4}\right)$, we have $c l_{Y}(\{e(d)\}) \subset c l_{Y}(\{e(a)\})$. Also, we know that there exists $y \in \bigcap\left\{c l_{Y}(\{e(d)\}): d \in D \cup\{x\}\right\}$, so $y \in \bigcap\left\{c l_{Y}(\{e(a)\}): a \in A \cup\{x\}\right\}$. Therefore, $\bigcap\left\{c_{Y}(\{e(a)\}): a \in A \cup\{x\}\right\} \neq \emptyset$, i.e., $A \in N_{e}^{S}(x)$.

To $\left(\operatorname{snf}_{2}\right)$ : Let $x \in L$. If $x=0$ then, by the definition, $N_{e}^{S}(0) \neq \emptyset$. If $x \in L \backslash\{0\}$, then $\{x\} \in N_{e}^{S}(x)$ because $e(x) \in c_{Y}(\{e(x)\})$. Consequently, ( $\left.\operatorname{snf}_{4}\right)$ is also valid.

To $\left(\operatorname{snf}_{3}\right)$ : By the definition, it is obvious.

To $\left(\operatorname{snf}_{5}\right)$ : Let $x, z \in L$ and $x \leq z$. Without loss of generality, let $x \neq 0$ and $A \in N_{e}^{S}(x)$. Then $\cap\left\{c l_{Y}(\{e(a)\}): a \in A \cup\{x\}\right\} \neq \emptyset$. By considering ( $\left.\mathrm{tfe}_{4}\right)$ and the assumption, we have $c l_{Y}(\{e(x)\}) \subset c l_{Y}(\{e(z)\})$. Therefore,

$$
\cap\left\{c l_{Y}(\{e(a)\}) \mid a \in A \cup\{z\}\right\} \neq \emptyset \text {, i.e., } A \in N_{e}^{S}(z) .
$$

To $\left(\operatorname{snf}_{6}\right)$ : Without loss of generality, let $x \in L \backslash\{0\}$ and $A \vee D \in N_{e}^{S}(x)$. Then $\cap\left\{c l_{Y}(\{e(z)\}): z \in(A \vee D) \cup\{x\}\right\} \neq \emptyset$. So, there exists $y \in \cap\left\{c l_{Y}(\{e(z)\}): z \in\right.$ $(A \vee D) \cup\{x\}\}$, therefore, for all $a \in A$ and all $d \in D$, we have $y \in c l_{Y}(\{e(a \vee d)\})$ 
which, by (tfe5), implies that, for all $a \in A$ and all $d \in D, y \in c l_{Y}(\{e(a)\}) \cup$ $c l_{Y}(\{e(d)\})$. If for $a_{1} \in A, y \notin c l_{Y}\left(\left\{e\left(a_{1}\right)\right\}\right)$ and if for $d_{1} \in D, y \notin c l_{Y}\left(\left\{e\left(d_{1}\right)\right\}\right)$, then we have $y \notin c l_{Y}\left(\left\{e\left(a_{1}\right)\right\}\right) \cup c l_{Y}\left(\left\{e\left(d_{1}\right)\right\}\right)$, which is a contradiction. So, either, for all $a \in A, y \in c l_{Y}(\{e(a)\})$ or, for all $d \in D, y \in c l_{Y}(\{e(d)\})$. Therefore, either $y \in \cap\left\{c l_{Y}(\{e(z)\}): z \in A \cup\{x\}\right\}$ or $y \in \cap\left\{c l_{Y}(\{e(z)\}): z \in D \cup\{x\}\right\}$, i.e., either $A \in N_{e}^{S}(x)$ or $D \in N_{e}^{S}(x)$.

To $\left(\operatorname{snf}_{7}\right)$ : Without loss of generality, let $x \in L \backslash\{0\}, A \subset L$ and $\left\{\bar{z}^{N_{e}^{S}} \mid z \in A\right\} \in$ $N_{e}^{S}(x)$. Then there exists $y \in c l_{Y}(\{e(x)\})$ such that, for all $z \in A, y \in$ $c l_{Y}\left(\left\{e\left(\bar{z}^{N_{e}^{S}}\right)\right\}\right)$. So, for all $z \in A$, we have, $y \in c l_{Y}\left(\left\{e\left(z^{c}\right)\right\}\right)$. According to $\left(\mathrm{tfe}_{3}\right)$, $e\left(z^{c}\right) \in c l_{Y}(\{e(z)\})$ so $c l_{Y}\left(\left\{e\left(z^{c}\right)\right\}\right) \subset c l_{Y}(\{e(z)\})$. Therefore, for all $z \in A$, we have $y \in c l_{Y}(\{e(z)\})$. Hence, by considering $y \in c l_{Y}(\{e(x)\})$, we have $A \in N_{e}^{S}(x)$.

To (sym): Without loss of generality, let $x \in L \backslash\{0\}$ and $A \in N_{e}^{S}(x)$. Then there exists $y \in \operatorname{cl}_{Y}(\{e(x)\})$ such that $y \in \cap\left\{c l_{Y}(\{e(a)\}) \mid a \in A\right\}$. Hence, $\{x\} \cup A \in$ $N_{e}^{S}(x)$. Now let $z$ be an arbitrary element of $A$, so $y \in c l_{Y}(\{e(z)\}) \cap c l_{Y}(\{e(x)\}) \cap$ $\left(\cap\left\{c l_{Y}(\{e(a)\}) \mid a \in A\right\}\right)$. Then $c l_{Y}(\{e(z)\}) \cap\left(\cap\left\{c l_{Y}(\{e(b)\}) \mid b \in\{x\} \cup A\right\} \neq \emptyset\right.$, i.e., $\{x\} \cup A \in N_{e}^{S}(z)$.

Definition 4.5. Let $(L, N)$ be a supernear frame and $x \in L \backslash\{0\}$. Then, $G \subset L$ is called an $x$-frame clan in $N$ if and only if it satisfies the following conditions:

$\left(\right.$ fcla $\left._{0}\right) 0 \notin G$;

(fcla 1$) z_{1} \in G$ and $z_{1} \leq z_{2} \in L$ imply $z_{2} \in G$;

$($ fcla $) z_{1}, z_{2} \in L$ and $z_{1} \vee z_{2} \in G$ imply $z_{1} \in G$ or $z_{2} \in G$;

$\left(\right.$ fcla $\left._{3}\right) x \in G \in N(x)$;

(fcla 4$) y \in L$ and $\bar{y}^{N} \in G$ imply $y \in G$.

The set of all $x$-frame clans in $N$ is denoted by $F C L A(L)$.

Remark 4.6. Let $(L, N)$ be a supernear frame and $x \in L \backslash\{0\}$. Then, $x_{N}:=$ $\left\{z \in L \mid x \leq \bar{z}^{N}\right\}$ is a frame clan in $N$, which is maximal in $N(x)$ with respect to inclusion.

Proof. First we show that $x_{N}$ satisfies $\left(\right.$ fcla $\left._{0}\right)$ to (fcla4).

To $\left(\mathrm{fcla}_{0}\right)$ : Evident.

To (fcla 1 ): Let $z_{1} \in x_{N}$ and $z_{1} \leq z_{2} \in L$. So, $x \leq{\overline{z_{1}}}^{N} \leq{\overline{z_{2}}}^{N}$, therefore, $z_{2} \in x_{N}$.

To (fcla 3 ): Without loss of generality, let $x \in L \backslash\{0\}$. Evidently, $x \in x_{N}$. Now we set $A:=\left\{\bar{z}^{N}: z \in x_{N}\right\}$, hence $A<<\{x\}$ and, by $\left(\operatorname{snf}_{4}\right),\{x\} \in N(x)$. Therefore, by $\left(\operatorname{snf}_{1}\right), A \in N(x)$, which, by $\left(\operatorname{snf}_{7}\right)$, implies $x_{N} \in N(x)$.

To (fcla $)$ : Without loss of generality, let $z_{1}, z_{2} \in L \backslash\{0,1\}$ with $z_{1} \vee z_{2} \in$ $x_{N}$. Since $x_{N} \in N(x)$, according to $\left(\operatorname{snf}_{1}\right)$, we have $\left\{z_{1} \vee z_{2}\right\} \in N(x)$, hence $\left\{z_{1}\right\} \vee\left\{z_{2}\right\} \in N(x)$ and by $\left(\operatorname{snf}_{6}\right)$, either $\left\{z_{1}\right\} \in N(x)$ or $\left\{z_{2}\right\} \in N(x)$. Therefore, by the definition of N-closure, $x \leq \bar{z}_{1}^{N}$ or $x \leq \bar{z}_{2}^{N}$, i.e., $z_{1} \in x_{N}$ or $z_{2} \in x_{N}$.

To $\left(\right.$ fcla $\left._{4}\right)$ : Let $y \in L$ and $\bar{y}^{N} \in x_{N}$. So, $x \leq{\overline{\left(\bar{y}^{N}\right)}}^{N} \leq \bar{y}^{N}$, hence $y \in x_{N}$. Therefore, $N(x)$ is an $x$-frame clan in $N$. Now let $A \in N(x)$ with $x_{N} \subset A$. Without loss of generality, for $z \in A \backslash\{1\}$, we have $\{z\} \in N(x)$, hence $x \leq \bar{z}^{N}$, which implies $z \in x_{N}$. Consequently, $A=x_{N}$. 
Definition 4.7. A supernear frame $(L, N)$ is called a superclan frame if and only if it satisfies the following condition:

(cla) $x \in L \backslash\{0\}$ and $A \in N(x)$ imply $\exists C \in F C L A(L) \cap N(x)$ such that $A \subset C$.

Remark 4.8. A paranear frame which is a superclan frame is called a paraclan frame. And the corresponding category is denoted by CLA-PNFRM - it is a full subcategory of PNFRM.

Example 4.9. Let $(e, L, Y)$ be a symmetric topological frame extension. Then the paranear frame $\left(L, N_{e}^{S}\right)$, in Lemma 4.4 , is a paraclan.

Proof. We show that $\left(L, N_{e}^{S}\right)$ satisfies (cla). Let $x \in L \backslash\{0\}$ and $A \in N_{e}^{S}(x)$. Then there exists $y \in c l_{Y}(\{e(x)\})$ such that $y \in \cap\left\{c l_{Y}(\{e(a)\}) \mid a \in A\right\}$. We put $C_{y}:=\left\{z \in L \backslash\{0\} \mid y \in c l_{Y}(\{e(z)\})\right\}$ and we show that $C_{y} \in F C L A(L)$.

To $\left(\mathrm{fcla}_{0}\right)$ : Evident.

To (fcla $)$ : Let $z_{1} \in C_{y}$ and $z_{1} \leq z_{2} \in L$. According to $\left(\mathrm{tfe}_{4}\right)$ and the definition of $C_{y}$, we have $y \in c l_{Y}\left(\left\{e\left(z_{1}\right)\right\}\right) \subset c l_{Y}\left(\left\{e\left(z_{2}\right)\right\}\right)$. Hence, $z_{2} \in C_{y}$.

To $\left(\right.$ fcla $\left._{2}\right)$ : Let $z_{1}, z_{2} \in L$ and $z_{1} \vee z_{2} \in C_{y}$. So, $y \in c l_{Y}\left(\left\{e\left(z_{1} \vee z_{2}\right)\right\}\right)$ and, according to $\left(\mathrm{tfe}_{5}\right)$, we have $c l_{Y}\left(\left\{e\left(z_{1} \vee z_{2}\right)\right\}\right) \subset c l_{Y}\left(\left\{e\left(z_{1}\right)\right\}\right) \cup c l_{Y}\left(\left\{e\left(z_{2}\right)\right\}\right)$. Therefore, $y \in c l_{Y}\left(\left\{e\left(z_{1}\right)\right\}\right)$ or $y \in c l_{Y}\left(\left\{e\left(z_{2}\right)\right\}\right)$. Hence, $z_{1} \in C_{y}$ or $z_{2} \in C_{y}$.

To (fcla 3 ): We know that $y \in c l_{Y}(\{e(x)\})$ so $x \in C_{y}$. Also, we know that, for all $z \in C_{y}, y \in \operatorname{cl}_{Y}(\{e(z)\})$. So, $C_{y} \in N_{e}^{S}(x)$.

To $\left(\right.$ fcla $\left._{4}\right)$ : Let $z \in L$ with $\bar{z}^{N_{e}^{S}} \in C_{y}$. So, $y \in c l_{Y}\left(\left\{e\left(\bar{z}^{N_{e}^{S}}\right)\right\}\right)$ and, by Lemma $4.4,{ }^{-N_{e}^{S}}=c$, hence $y \in \operatorname{cl}_{Y}\left(\left\{e\left(z^{c}\right)\right\}\right)$.

On the other hand, according to $\left(\mathrm{tfe}_{3}\right)$, we have $e\left(z^{c}\right) \in c l_{Y}(\{e(z)\})$ and, because $c l_{Y}$ is a topological closure operator, $c l_{Y}\left(\left\{e\left(z^{c}\right)\right\}\right) \subset c l_{Y}(\{e(z)\})$. Consequently, $y \in \operatorname{cl}_{Y}(\{e(z)\})$, so $z \in C_{y}$. Therefore, $C_{y} \in F C L A(L)$.

Now let $a \in A \backslash\{0\}$. Then $y \in \operatorname{cl}_{Y}(\{e(a)\})$, i.e., $a \in C_{y}$. Therefore, $A \backslash\{0\} \subset$ $C_{y}$. This implies $\left(L, N_{e}^{S}\right)$ is a paraclan.

Theorem 4.10. Let $F$ be defined on SYMTFE by setting the following:

(a) For a symmetric topological frame extension $(e, L, Y)$ we put $F(e, L, Y):=$ $\left(L, N_{e}^{S}\right)$;

(b) For a tfe-morphism $(g, h):(e, L, Y) \rightarrow\left(e^{\prime}, L^{\prime}, Y^{\prime}\right)$ we put $F(g, h):=g$. Then, $F$ is a functor from SYMTFE to CLA-PNFRM.

Proof. According to Example 4.9 the only remaining fact is to show that $g:\left(L, N_{e}^{S}\right) \rightarrow\left(L^{\prime}, N_{e^{\prime}}^{S}\right)$ is an snf-map. So, let $x \in L \backslash\{0\}$ and $A \in N_{e}^{S}(x)$. Then $y \in \cap\left\{c l_{Y}(\{e(a)\}) \mid a \in A\right\}$ for some $y \in c l_{Y}(\{e(x)\})$. Since $h$ is continuous we have $h(y) \in c l_{Y^{\prime}}(\{h(e(x))\})$. Also, since the diagram in Definition 4.1 commutes, $c l_{Y^{\prime}}(\{h(e(x))\})=c l_{Y^{\prime}}\left(\left\{e^{\prime}(g(x))\right\}\right)$. Therefore, $h(y) \in c l_{Y^{\prime}}\left(\left\{e^{\prime}(g(x))\right\}\right)$. On the other hand, for $a \in A$, we have $y \in c l_{Y}(\{e(a)\})$ and, consequently, $h(y) \in c l_{Y^{\prime}}(\{h(e(a))\})=c l_{Y^{\prime}}\left(\left\{e^{\prime}(g(a))\right\}\right)$. Therefore, this all implies $g[A] \in$ $N_{e^{\prime}}^{S}(g(x))$.

\section{Strict topological FRAme EXtension}

In the previous section, we have found a functor from SYMTFE to CLAPNFRM. Now, we are going to introduce a related one in the opposite direction. 
Lemma 5.1. Let $(L, N)$ be a paranear frame and $L^{*}:=F C L A(L) \cup\{\emptyset\}$. Let the operator $c_{L^{*}}: P L^{*} \rightarrow P L^{*}$ be defined by $c_{L^{*}}\left(A^{*}\right):=\left\{D \in L^{*} \mid \Delta A^{*} \subset D\right\}$, where $A^{*} \subset L^{*}$ and $\Delta A^{*}:=\left\{z \in L \mid \forall F \in A^{*} z \in F\right\}$ (by convention, if $A^{*}=\emptyset$, then $\Delta A^{*}=L$ and if $\emptyset \in A^{*}$, then $\Delta A^{*}=\emptyset$ ). Then, $c_{L^{*}}$ is a topological closure operator.

Proof. We show that $c l_{L^{*}}$ satisfies all conditions of a topological closure operator.

(1) Suppose $c l_{L^{*}}(\emptyset) \neq \emptyset$. Then there exists $D \in L^{*}$ such that $L=\Delta \emptyset \subset D$. Consequently, $0 \in D$, which leads us to a contradiction.

(2) Let $D \in A^{*}$ and $z \in \Delta A^{*}$, so that $z \in D$. Hence, $\Delta A^{*} \subset D$, therefore, $D \in c l_{L^{*}}\left(A^{*}\right)$ which implies $A^{*} \subset c l_{L^{*}}\left(A^{*}\right)$.

(3) Let $A_{1}^{*} \subset A_{2}^{*} \subset L^{*}$ and $D \in \operatorname{cl}_{L^{*}}\left(A_{1}^{*}\right)$, so that $\Delta A_{1}^{*} \subset D$. Also, $\Delta A_{2}^{*} \subset \Delta A_{1}^{*}$, therefore, $D \in c l_{L^{*}}\left(A_{2}^{*}\right)$ which implies $c l_{L^{*}}\left(A_{1}^{*}\right) \subset c l_{L^{*}}\left(A_{2}^{*}\right)$.

(4) Let $A_{1}^{*}, A_{2}^{*} \subset L^{*}$ and $D \in c l_{L^{*}}\left(A_{1}^{*} \cup A_{2}^{*}\right)$. Suppose $D \notin c l_{L^{*}}\left(A_{1}^{*}\right) \cup c l_{L^{*}}\left(A_{2}^{*}\right)$, so we can choose $z_{1} \in \Delta A_{1}^{*}$ such that $z_{1} \notin D$ and $z_{2} \in \Delta A_{2}^{*}$ such that $z_{2} \notin D$. By the assumption, $\Delta\left(A_{1}^{*} \cup A_{2}^{*}\right) \subset D$ so $z_{1} \vee z_{2} \in \Delta\left(A_{1}^{*} \cup A_{2}^{*}\right)$, because for $F \in\left(A_{1}^{*} \cup A_{2}^{*}\right)$ either $F \in A_{1}^{*}$ or $F \in A_{2}^{*}$. If $F \in A_{1}^{*}$, then $z_{1} \in F$, hence $\emptyset \neq F \in F C L A(L)$ with $z_{1} \leq z_{1} \vee z_{2}$. And $F$ satisfies $\left(\right.$ fcla $_{1}$ ), so $z_{1} \vee z_{2} \in F$. If $F \in A_{2}^{*}$, then $z_{2} \in F$, hence $\emptyset \neq F \in F C L A(L)$ with $z_{2} \leq z_{1} \vee z_{2}$. And $F$ satisfies (fcla 1 ), so $z_{1} \vee z_{2} \in F$. Therefore, $z_{1} \vee z_{2} \in \Delta\left(A_{1}^{*} \cup A_{2}^{*}\right) \subset D$. Consequently, since $D$ satisfies $\left(\right.$ fcla $\left._{2}\right)$, we have $z_{1} \in D$ or $z_{2} \in D$. This leads us to a contradiction. Therefore, $c l_{L^{*}}\left(A_{1}^{*} \cup A_{2}^{*}\right) \subset c l_{L^{*}}\left(A_{1}^{*}\right) \cup c l_{L^{*}}\left(A_{2}^{*}\right)$.

(5) Let $D \in c l_{L^{*}}\left(c l_{L^{*}}\left(A^{*}\right)\right)$ and suppose $D \notin c l_{L^{*}}\left(A^{*}\right)$. Then there exists $z \in \Delta A^{*}$ such that $z \notin D$. On the other hand, by assumption, $\Delta c l_{L^{*}}\left(A^{*}\right) \subset D$. Also, we know that, for arbitrary $x \in \Delta A^{*}, F \in c l_{L^{*}}\left(A^{*}\right)$ implies $\Delta A^{*} \subset F$. Hence, $x \in F$, which proves $\Delta A^{*} \subset \Delta c l_{L^{*}}\left(A^{*}\right)$. Consequently, we have $z \in D$, which is a contradiction. Therefore, $c l_{L^{*}}\left(c l_{L^{*}}\left(A^{*}\right)\right) \subset c l_{L^{*}}\left(A^{*}\right)$ (our proving is based essentially on the fact that $\emptyset \notin A^{*} \subset L^{*}$, but this does not matter).

Theorem 5.2. Let $(L, N)$ and $(Y, M)$ be paranear frames and $f: L \rightarrow Y$ be an snf-map. Let the function $f^{*}: L^{*} \rightarrow Y^{*}$ be defined by setting:

$$
f^{*}(C):= \begin{cases}\emptyset & \text { if } C=\emptyset \\ \left\{y \in Y \mid \exists z \in f[C] \text { s.t. } z \leq \bar{y}^{M}\right\} & \text { otherwise. }\end{cases}
$$

Then, the following statements are valid:

(1) $f^{*}$ is a continuous map from $\left(L^{*}, c l_{L^{*}}\right)$ to $\left(Y^{*}, c l_{Y^{*}}\right)$;

(2) The composites $f^{*} \circ e_{N}$ and $e_{M} \circ f$ coincide, where $e_{N}: L \rightarrow L^{*}$ is defined by setting:

$$
e_{N}(x):= \begin{cases}\emptyset & \text { if } x=0 \\ x_{N} & \text { otherwise. }\end{cases}
$$

Proof. First we show that $f^{*}(C) \in Y^{*}$. If $C=\emptyset$, then $f^{*}(C)=\emptyset$, therefore, $f^{*}(C) \in Y^{*}$. Now let $C \neq \emptyset$. We show that $f^{*}(C) \in F C L A(Y)$.

To $\left(\right.$ fcla $\left._{0}\right)$ : Suppose $0 \in f^{*}(C)$. Then there exists $z \in f[C]$ with $z \leq \overline{0}^{M}=$ 0 , therefore, $0 \in f[C]$. Since $f$ is a frame homomorphism, $0 \in C$, which is a contradiction to $C \in F C L A(L)$. 
To $\left(\right.$ fcla $\left._{1}\right)$ : Let $y_{1} \in f^{*}(C)$ and $y_{1} \leq y_{2} \in Y$. Then there exists $z \in f[C]$ with $z \leq{\overline{y_{1}}}^{M} \leq{\overline{y_{2}}}^{M}$ so $y_{2} \in f^{*}(C)$.

To $\left(\right.$ fcla $_{2}$ ): Let $y_{1}, y_{2} \in Y$ and $y_{1} \vee y_{2} \in f^{*}(C)$. Then $y_{1} \vee y_{2} \neq 0$, so either $y_{1} \neq 0$ or $y_{2} \neq 0$. If $y_{1} \neq 0$ and $y_{2}=0$, then $y_{1}=y_{1} \vee y_{2} \in f^{*}(C)$ and, analogously, if $y_{1}=0$ and $y_{2} \neq 0$, then $y_{2}=y_{1} \vee y_{2} \in f^{*}(C)$. Now, suppose $y_{1} \neq 0 \neq y_{2}$. Then, for some $z \in f[C]$, we have $z \leq{\overline{y_{1} \vee y_{2}}}^{M}$. By the hypothesis, $z \neq 0$, so, $\{z\} \in M(z)$. Also, we have $\left\{{\overline{y_{1}}}^{M} \vee{\overline{y_{2}}}^{M}\right\}<<\{z\}$. Hence, by $\left(\operatorname{snf}_{1}\right),\left\{{\overline{y_{1}}}^{M} \vee \bar{y}_{2}{ }^{M}\right\} \in M(z)$ and by $\left(\operatorname{snf}_{6}\right),\left\{{\overline{y_{1}}}^{M}\right\} \in M(z)$ or $\left\{{\overline{y_{2}}}^{M}\right\} \in M(z)$. Therefore, by $\left(\operatorname{snf}_{7}\right),\left\{y_{1}\right\} \in M(z)$ or $\left\{y_{2}\right\} \in M(z)$, which proves that $z \leq{\overline{y_{1}}}^{M}$ or $z \leq{\overline{y_{2}}}^{M}$. Therefore, $y_{1} \in f^{*}(C)$ or $y_{2} \in f^{*}(C)$.

To (fcla $)$ : There exists $x \in L$ such that $x \in C \in N(x)$, hence $1 \in C$ and, because $f$ is a frame homomorphism, $f(1)=1=\overline{1}^{M}$. Therefore, $1 \in f^{*}(C)$. On the other hand, since $f$ is snf-map, $C \in N(x)$ implies $f[C] \in M(f(x))$. Also, $M(f(x)) \subset M(1)$, therefore, $f[C] \in M(1)$. We put $A:=\left\{\bar{y}^{M} \mid y \in f^{*}(C)\right\}$. Then, for $y \in f^{*}(C)$, we have $z_{y} \leq \bar{y}^{M}$ for some $z_{y} \in f[C]$. By the hypothesis, $z_{y} \neq 0$ and $A<<\left\{z_{y} \mid y \in f^{*}(C)\right\} \subset f[C] \in M(1)$. Therefore, by $\left(\operatorname{snf}_{1}\right)$, we have $A \in M(1)$. Consequently, by $\left(\operatorname{snf}_{7}\right)$ we can say $f^{*}(C) \in M(1)$.

To $\left(\right.$ fcla $\left._{4}\right)$ : Let $y \in Y$ such that $\bar{y}^{M} \in f^{*}(C)$. Then, we have $z \leq{\overline{\left(\bar{y}^{M}\right)}}^{M}$ for some $z \in f[C]$, hence by knowing ${\overline{\left(\bar{y}^{M}\right)}}^{M} \leq \bar{y}^{M}$, we have $z \leq \bar{y}^{M}$, which implies $y \in f^{*}(C)$.

All together show that now $f^{*}(C) \in F C L A(Y)$, hence, for every $C \in L^{*}$, we get $F^{*}(C) \in Y^{*}$.

To (1): We show that $f^{*}$ is a continuous map. Let $A^{*} \subset L^{*}$ and $C \in \operatorname{cl}_{L^{*}}\left(A^{*}\right)$. We have to verify $f^{*}(C) \in \operatorname{cl}_{Y^{*}}\left(f^{*}\left[A^{*}\right]\right)$.

Case 1: If $A^{*}=\emptyset$, then the above implication is valid.

Case 2: Let $\emptyset \in A^{*}$. Then $\Delta A^{*}=\emptyset$, and $c l_{L^{*}}\left(A^{*}\right)=L^{*}$, which implies $f^{*}\left[c l_{L^{*}}\left(A^{*}\right)\right]$

$=f^{*}\left[L^{*}\right] \subset Y^{*}$ and, since $\emptyset \in f^{*}\left[A^{*}\right]$, we have $c l_{Y^{*}}\left[f^{*}\left[A^{*}\right]\right]=Y$. Therefore, $f^{*}\left[c l_{L^{*}}\left(A^{*}\right)\right] \subset c l_{Y^{*}}\left[f^{*}\left[A^{*}\right]\right]$.

Case 3: If $f^{*}(C)=\emptyset$, then $\emptyset \in f^{*}\left[A^{*}\right]$ and $c l_{Y^{*}}\left(f^{*}\left[A^{*}\right]\right)=Y^{*}$, consequently, $f^{*}(C) \in \operatorname{cl}_{Y^{*}}\left(f^{*}\left[A^{*}\right]\right)$.

Case 4: For every $D \in A^{*}$, we have $D \neq \emptyset$. Suppose $f^{*}(C) \notin c l_{Y^{*}}\left(f^{*}\left[A^{*}\right]\right)$. Then there exists $y \in \Delta f^{*}\left[A^{*}\right], y \notin f^{*}(C)$. We put $A_{y}:=\left\{x \in L \mid f(x) \leq \bar{y}^{M}\right\}$. Then $\vee A_{y} \notin C$. If $\vee A_{y} \in C$, we have $f\left[\vee A_{y}\right]=\vee\left\{f(a) \mid a \in A_{y}\right\}=\vee f\left[A_{y}\right] \in$ $f[C] \backslash\{0\}$. Consequently, $f\left[\vee A_{y}\right] \leq \bar{y}^{M}$, therefore, $y \in f^{*}(C)$, which is a contradiction. Therefore, $\vee A_{y} \notin C$. By the hypothesis, $C \in \operatorname{cl}_{L^{*}}\left(A^{*}\right)$ so $\Delta A^{*} \subset C$. Therefore, $\vee A_{y} \notin \Delta A^{*}$. Hence, there exists $D \in A^{*}$ such that $\vee A_{y} \notin D$. Consequently, $f^{*}(D) \in f^{*}\left[A^{*}\right]$ and since $y \in \Delta f^{*}\left[A^{*}\right]$ we have $y \in f^{*}(D)$.

Now, by definition of $f^{*}$, there exists $z \in f[D]$ such that $z \leq \bar{y}^{M}$. But $z=f(d)$ for some $d \in D$, hence $d \in A_{y}$ and $d \leq \vee A_{y}$. Therefore, $\vee A_{y} \in D$, which is a contradiction. So, $f^{*}(C) \in c l_{Y^{*}}\left(f^{*}\left[A^{*}\right]\right)$. Therefore, we obtain that $f^{*}$ is a continuous map from $\left(L^{*}, c l_{L^{*}}\right)$ to $\left(Y^{*}, c l_{Y^{*}}\right)$.

To (2): If $x=0$, then, by considering $f$ is frame homomorphism, we have $e_{M}(f(x))=\emptyset=f^{*}\left(e_{N}(x)\right)$. If $x \neq 0$, then $f(x) \neq 0$ and $e_{M}(f(x))=f(x)_{M}$ with $0 \notin f(x)_{M}$ and $f(x)_{M} \in M(f(x)) \backslash\{\emptyset\}$. Firstly we show that $f^{*}\left(x_{N}\right) \in M(f(x))$. 
We know that $f^{*}\left(x_{N}\right) \neq \emptyset$. Now since $x_{N} \in N(x)$, we get $f\left[x_{N}\right] \in M(f(x))$. And we put $A:=\left\{\bar{y}^{M}: y \in f^{*}\left(x_{N}\right)\right\}$. For arbitrary $y \in f^{*}\left(x_{N}\right)$ there exists $z \in f\left[x_{N}\right]$ such that $z \leq \bar{y}^{M}$, hence $z=f(a)$ for some $a \in x_{N}$, and $x \leq \bar{a}^{N}$. Since $f$ is an snf-map, we have $f(x) \leq f\left(\bar{a}^{N}\right) \leq \overline{f(a)}^{M}=\bar{z}^{M} \leq{\overline{\left(\bar{y}^{M}\right)}}^{M} \leq \bar{y}^{M}$. So, $f(x) \leq \bar{y}^{M}$ and consequently, $A<<f\left[x_{N}\right]$. Then, by $\left(\operatorname{snf}_{1}\right), A \in M(f(x))$ and, by $\left(\operatorname{snf}_{7}\right)$, we have $f^{*}\left(x_{N}\right) \in M(f(x))$.

Secondly, we verify that $f(x)_{M} \subset f^{*}\left(x_{N}\right)$. Let $y \in f(x)_{M}$. Then $0 \neq f(x) \leq$ $\bar{y}^{M}$ and, consequently, $y \in f^{*}\left(x_{N}\right)$ so $f(x)_{M} \subset f^{*}\left(x_{N}\right)$. Now since $f(x)_{M}$ is maximal in $M(f(x)) \backslash\{\emptyset\}$, we have $f(x)_{M}=f^{*}\left(x_{N}\right)$.

Theorem 5.3. Let $G$ be defined as follows:

(a) $G(L, N):=\left(e_{N}, L, L^{*}\right)$ for any paraclan frame $(L, N)$ where $L:=\left(L,{ }^{-N}\right)$ and $L^{*}:=\left(L^{*}, c l_{L *}\right)$;

(b) $G(f):=\left(f, f^{*}\right)$ for any snf-map $f:(L, N) \rightarrow(Y, M)$.

Then, $G$ is a functor from CLA-PNFRM to SYMTFE.

Proof. We already know that $\left(L,^{-N}\right)$ is a Boolean frame with frame-closure ${ }^{-N}: L \rightarrow L, L^{*}:=\left(F C L A(L) \cup\{\emptyset\}, c l_{L^{*}}\right)$ is a topological space and $e_{N}: L \rightarrow L^{*}$ is a function. Now we verify $e_{N}$ satisfies axioms $\left(\mathrm{tfe}_{0}\right)$ to $\left(\mathrm{tfe}_{5}\right)$ and $(\mathrm{sym})$.

To $\left(\mathrm{tfe}_{0}\right)$ : By the definition, we know that $e_{N}(0)=\emptyset$.

To $\left(\mathrm{tfe}_{1}\right)$ : We have to show that $c l_{L^{*}}\left(e_{N}([L \backslash\{0\}])\right)=F C L A(L)$. Let $D \in$ $F C L A(L)$ and suppose $D \notin c l_{L^{*}}\left(e_{N}[L \backslash\{0\}]\right)$. Then $\Delta e_{N}[L \backslash\{0\}] \nsubseteq D$, so there exists $z \in \Delta e_{N}[L \backslash\{0\}]$ such that $z \notin D$. Consequently, $z=1$. On the other hand, there exists $x \in L \backslash\{0\}$ such that $x \in D$, therefore, $1 \in D$, which is a contradiction. Conversely, let $D \in c l_{L^{*}}\left(e_{N}[L \backslash\{0\}]\right)$, so $\Delta e_{N}[L \backslash\{0\}] \subset D$. Since $1 \in \Delta e_{N}[L \backslash\{0\}]$, we have $1 \in D$ and, consequently, $D \in F C L A(L)$. Note that $L^{*}:=F C L A(L) \cup\{\emptyset\}$ and $A \in e_{N}[L \backslash\{0\}]$ implies $A=e_{N}(x)$ for $x \in L \backslash\{0\}$. Also, since $x \leq \overline{1}^{N}$, we have $1 \in A$.

To $\left(\mathrm{tfe}_{2}\right)$ : For $z \in L \backslash\{0,1\}$ we put

$$
A_{z}:=\left\{x \in L \backslash\{0,1\}: e_{N}(x) \in \operatorname{cl}_{L^{*}}\left(\left\{e_{N}(z)\right\}\right)\right\} .
$$

And we claim $\bar{z}^{N}$ is upper bound of $A_{z}$. Let $x \in A_{z}$. Then $e_{N}(x) \in c l_{L^{*}}\left(\left\{e_{N}(z)\right\}\right)$, hence $\Delta\left\{z_{N}\right\}=\Delta\left\{e_{N}(z)\right\} \subset e_{N}(x)=x_{N}$. Consequently, $z \in x_{N}$, therefore, $x \leq$ $\bar{z}^{N}$, so $\vee e_{N}^{-1}\left[c l_{L^{*}}\left(\left\{e_{N}(z)\right\}\right)\right]=\vee A_{z} \leq \bar{z}^{N}$. Conversely, we claim $\vee A_{z}$ is an upper bound of $D:=\{y \in L \backslash\{0,1\} \mid\{z\} \in N(y)\}$. Let $y \in D$ so $\{z\} \in N(y)$ and $y \leq \bar{z}^{N}$. We have to verify $e_{N}(y) \in \operatorname{cl}_{L^{*}}\left(\left\{e_{N}(z)\right\}\right)$, which means $\Delta\left\{e_{N}(z)\right\} \subset e_{N}(y)$. Let $a \in \Delta\left\{e_{N}(z)\right\}$ so $a \in e_{N}(z)=z_{N}$. Then $z \leq \bar{a}^{N}$, so $y \leq \bar{z}^{N} \leq{\overline{\left(\bar{a}^{N}\right)}}^{N} \leq \bar{a}^{N}$, therefore, $a \in e_{N}(y)$. Consequently, $y \leq \vee \bar{A}_{z}$. But $\bar{z}^{N}$ is a join of $D$, hence $\bar{z}^{N} \leq \vee A_{z}$. All together imply $\bar{z}^{N}=\vee e_{N}^{-1}\left[c l_{L^{*}}\left(\left\{e_{N}(z)\right\}\right)\right]$.

To $\left(\operatorname{tfe}_{3}\right)$ : Let $z \in L$. If $z=0$, then $e_{N}\left(\bar{z}^{N}\right)=\emptyset$ and $c l_{L^{*}}\left(\left\{e_{N}(z)\right\}\right)=$ $c l_{L^{*}}(\{\emptyset\})=L^{*}$. Therefore, $e_{N}\left(\bar{z}^{N}\right) \subset c l_{L^{*}}\left(\left\{e_{N}(z)\right\}\right)$. Now, if $z \neq 0$, then $e_{N}(z)=$ $z_{N}$. We have to verify $\Delta\left\{z_{N}\right\} \subset\left(\bar{z}^{N}\right)_{N}$. Let $a \in \Delta\left\{z_{N}\right\}$. Then $z \leq \bar{a}^{N}$, hence $\bar{z}^{N} \leq{\overline{\left(\bar{a}^{N}\right)}}^{N} \leq \bar{a}^{N}$ which proves $a \in\left(\bar{z}^{N}\right)_{N}$. Therefore, $e_{N}\left(\bar{z}^{N}\right) \subset c l_{L^{*}}\left(\left\{e_{N}(z)\right\}\right)$.

To $\left(\operatorname{tfe}_{4}\right)$ : Let $a, b \in L$ such that $0 \neq a \leq b$ and $D \in c_{L^{*}}\left(\left\{e_{N}(a)\right\}\right)$. We have to verify $\Delta\left\{b_{N}\right\}=\Delta\left\{e_{N}(b)\right\} \subset D$. Let $z \in \Delta\left\{b_{N}\right\}$. Then $b \leq \bar{z}^{N}$ and, by the hypothesis, we get $a \leq \bar{z}^{N}$, so $z \in a_{N}=e_{N}(a)$. This implies $z \in D$. 
To $\left(\mathrm{tfe}_{5}\right)$ : Let $a, b \in L$ and, without loss of generality, let $a \neq 0 \neq b$. Suppose $D \notin$ $c l_{L^{*}}\left(\left\{e_{N}(a)\right\}\right) \cup c l_{L^{*}}\left(\left\{e_{N}(b)\right\}\right)$. Then $D \notin c l_{L^{*}}\left(\left\{e_{N}(a)\right\}\right)$ and $D \notin c l_{L^{*}}\left(\left\{e_{N}(b)\right\}\right)$. Then $\Delta\left\{a_{N}\right\}=\Delta\left\{e_{N}(a)\right\} \nsubseteq D$ and $\Delta\left\{b_{N}\right\}=\Delta\left\{e_{N}(b)\right\} \nsubseteq D$. Choose $x_{a} \in$ $a_{N}$ such that $x_{a} \notin D$ and $x_{b} \in b_{N}$ such that $x_{b} \notin D$, hence $a \leq \bar{x}_{a}^{N}$ and $b \leq{\overline{x_{b}}}^{N}$. We set $x:=x_{a} \vee x_{b}$, hence $x \in a_{N} \vee b_{N}$ and $x \notin D$, because $D \in$ $F C L A(L)$ or $D=\emptyset$. On the other hand, $x \in(a \vee b)_{N}=e_{N}(a \vee b)$. If $D \in$ $c l_{L^{*}}\left(\left\{e_{N}(a \vee b)\right\}\right)=c l_{L^{*}}\left(\left\{(a \vee b)_{N}\right\}\right)$, then $\Delta\left\{(a \vee b)_{N}\right\} \subset D$, therefore, $x \in D$, which is a contradiction.

To (sym): Let $x \in L \backslash\{0\}$ and $D \in \operatorname{cl}_{L^{*}}\left(\left\{e_{N}(x)\right\}\right)$. Then $\Delta\left\{e_{N}(x)\right\} \subset D$, hence $x_{N}=e_{N}(x) \subset D$. On the other hand, for some $y \in L \backslash\{0\}$ we have, $D \in N(y)$. Consequently, $\{y\} \cup D \in N(x)$ because, by the hypothesis, $(L, N)$ is paraclan frame. So, $D \in N(x) \backslash\{\emptyset\}$ and, because $e_{N}(x)$ is maximal in $N(x) \backslash\{\emptyset\}$, $e_{N}(x)=D$. Consequently, $e_{N}(x) \in c l_{L^{*}}(\{D\})$.

Therefore, $\left(e_{N}, L, L^{*}\right)$ is a symmetric topological frame extension. By Theorem 5.2 , we already know that $G(f)$ is a tfe-morphism. It is clear that, for snf-maps $(L, N) \stackrel{f}{\rightarrow}(Y, M) \stackrel{g}{\rightarrow}(Z, P)$, the equation $G(g \circ f)=G(g) \circ G(f)$ holds.

Corollary 5.4. The image of $G$ is contained in STRTFE.

Proof. Let $A^{*}$ be closed in $F C L A(L)$ and $D \in F C L A(L)$ be such that $D \notin A^{*}$. Then $D \notin c l_{L^{*}}\left(A^{*}\right)$, hence $\Delta A^{*} \nsubseteq D$. Consequently, there exists $z \in \Delta A^{*}$ such that $z \notin D$. Therefore, for each $F \in A^{*}$, we have $z \in F$. Now let $a$ be an arbitrary element of $\Delta\left\{e_{N}(z)\right\}$. Then $a \in z_{N}$ and, consequently, $z \leq \bar{a}^{N}$. Since $z \in F$ and $F \in F C L A(L)$, we have $\bar{a}^{N} \in F$ and, by (fcla 4$), a \in F$. Therefore, $\Delta\left\{e_{N}(z)\right\} \subset F$, thus $F \in c l_{L^{*}}\left(\left\{e_{N}(z)\right\}\right)$. And by considering $F$ is an arbitrary element of $A^{*}$, we have $A^{*} \subset c l_{L^{*}}\left(\left\{e_{N}(z)\right\}\right)$. On the other hand, since $z \notin D$, we have $\Delta\left\{e_{N}(z)\right\} \nsubseteq D$, so $D \notin c l_{L^{*}}\left(\left\{e_{N}(z)\right\}\right)$, which implies $c l_{L^{*}}\left(\left\{e_{N}(z)\right\}\right) \subset A^{*}$. Therefore, $A^{*}=\operatorname{cl}_{L^{*}}\left(\left\{e_{N}(z)\right\}\right)=\operatorname{cl}_{L^{*}}\left(e_{N}[\{z\}]\right)$. In the case that $A^{*}=F C L A(L)$ we have $A^{*}=\operatorname{cl}_{L^{*}}\left(e_{N}[L \backslash\{0\}]\right)$.

Theorem 5.5. Let $F:$ SYMTFE $\rightarrow$ CLA-PNFRM and $G:$ CLA-PNFRM $\rightarrow$ SYMTFE be the above defined functors. For each object $(L, N)$ of CLAPNFRM, let $t_{(L, N)}$ denote the identity map $i d_{L}: F(G(L, N)) \rightarrow(L, N)$. Then, $t: F \circ G \rightarrow 1_{\text {CLA-PNFRM }}$ is a natural equivalence from $F \circ G$ to the identity functor $1_{\text {CLA-PNFRM }}$

$$
\begin{array}{cc}
F(G(L, N)) \stackrel{i d_{L}}{\longrightarrow}(L, N) \\
F(G(f)) \downarrow & \downarrow f \\
\left.F(G(Y, M)) \stackrel{i d_{Y}}{\longrightarrow}(Y, M)\right) .
\end{array}
$$

I.e., $i d_{L}$ is a snf-map in both directions for each object $(L, N)$ and the above diagram commutes for each snf-map $f:(L, N) \rightarrow(Y, M)$.

Proof. The commutativity of the diagram is obvious because $F(G(f))=f$. It remains to prove that $i d_{L}: F(G(L, N)) \rightarrow(L, N)$ is an snf-map in both directions. Let $F(G(L, N))=\left(L, N_{1}\right)$. It suffices to show that, for each $x \in L \backslash\{0\}$, we have $N_{1}(x) \subset N(x) \subset N_{1}(x)$. To this end, we assume $A \in N_{1}(x)$. Then there exists $C \in$ $c l_{L^{*}}\left(\left\{e_{N}(x)\right\}\right)$ such that $C \in \bigcap\left\{c l_{L^{*}}\left(\left\{e_{N}(a)\right\}\right): a \in A\right\}$, hence $\Delta\left\{e_{N}(x)\right\} \subset C$. 
We get $x \in C$ and $C \in N(z)$ for some $z \in L \backslash\{0\}$. Since $N$ is symmetric, we have $\{z\} \cup C \in N(x)$, which implies $C \in N(x)$ by $\left(\operatorname{snf}_{1}\right)$. Let $a$ be an arbitrary element of $A$. Then $C \in c l_{L^{*}}\left(\left\{e_{N}(a)\right\}\right)$, hence $\Delta\left\{e_{N}(a)\right\} \subset C$, so $a \in C$. Consequently, by $\left(\operatorname{snf}_{1}\right), A \in N(x)$. Conversely, let $A \in N(x)$. Since $(L, N)$ is a paraclan frame, we can choose an $x$-frame clan $C$ in $N$ with $A \subset C$. In order to show $A \in N_{1}(x)$, we need to verify

(i) $C \in \operatorname{cl}_{L^{*}}\left(\left\{e_{N}(x)\right\}\right)$;

(ii) $a \in A$ implies $C \in \operatorname{cl}_{L^{*}}\left(\left\{e_{N}(a)\right\}\right)$.

To (i): By the definition, it suffices to establish $\Delta\left\{e_{N}(x)\right\} \subset C$. Let $z \in$ $\Delta\left\{e_{N}(x)\right\}$. Then $x \leq \bar{z}^{N}$, hence $\bar{z}^{N} \in C$, so $z \in C$.

To (ii): Let $a$ be an element of $A$ and $z$ be an element of $\Delta\left\{e_{N}(a)\right\}$. Then $a \leq \bar{z}^{N}$. Also, since $a \in C$, we have $\bar{z}^{N} \in C$, so $z \in C$, which proves $C \in$ $c l_{L^{*}}\left(\left\{e_{N}(a)\right\}\right)$.

Remark 5.6. To make the last theorem more transparent, we claim that a paranear frame $(L, N)$ has a symmetric strict topological frame extension if and only if it is a paraclan frame. Hence, there exists a topological space $\left(Y, c l_{Y}\right)$ in which $L$ can be embedded and whose underlying set is the union of a set $X$ with some point not contained in $X$, so the $x$-near sets in $N$ are characterized by the fact that all of their closures meet in $Y$. We note that Boolean frames, hence uniform frames, or proximal frames, respectively, can be equivalently described in terms of supernear frames or paranear frames, respectively. Hence, for all the above mentioned and subsumed spaces, this fundamental theorem can also be applied.

Definition 5.7. A supernear frame $(L, N)$ is called separated if and only if it satisfies the following condition:

(sep) $x, z \in L \backslash\{0\}$ and $\{z\} \in N(x)$ imply $x=z$.

Remark 5.8. Let $(L, N)$ be a separated supernear frame. Then, $e_{N}: L \rightarrow L^{*}$ is injective.

Remark 5.9. Let $(e, L, Y)$ be a topological frame extension, where $e$ is injective and $Y=\left(Y, c l_{Y}\right)$ is a $\mathrm{T}_{1}$-space. Then $(L, N)$ is separated supernear frame.

\section{REFERENCES}

[1] B. Banaschewski, Extensions of topological spaces, Can. Math. Bull. 7 (1964), 1-23. DOI: 10.4153/CMB-1964-001-5

[2] H. L. Bentley, Nearness spaces and extension of topological spaces, in: Studies in Topology, Academic Press, NY, 1975, 47-66.

[3] D. Doitchinov, On a single theory for topological, proximity and uniform spaces (in Russian), Dokl. Akad. Nauk SSSR 156 (1964), 21-24.

[4] H. Herrlich, A concept of nearness, Gen. Topol. Appl. 4 (1974), 191-212. DOI: 10.1016/0016$660 \mathrm{X}(74) 90021-\mathrm{X}$

[5] H. Herrlich, Topological structures, Math. Centre Tracts 52 (1974), 59-122.

[6] V.M. Ivanova and A. Ivanov, Contiguity spaces and bicompact extensions of topological spaces (in Russian), Dokl. Akad. Nauk SSSR 127 (1959), 20-22.

[7] P. T. Johnstone, Stone Spaces, Cambridge Studies in Advanced Mathematics 3, Cambridge University Press, 1982.

[8] D. Leseberg, Supernearness, a common concept of supertopologies and nearness, Topol. Appl. 123 (2002), 145-165. DOI: 10.1016/S0166-8641(01)00178-X 
[9] D. Leseberg, Symmetrical extensions and generalized nearness, Note Mat. 22 (2003), 93111.

[10] D. Leseberg, Improved nearness research II, Rostock. Math. Kolloq. 66 (2011), 87-102.

[11] D. Leseberg, Improved nearness research III, Int. J. Math. Sci. Appl. 1 (2011), 939-952.

[12] Y. M. Smirnov, On the completeness of proximity spaces (in Russian), Dokl. Akad. Nauk SSSR 88 (1953), 761-794.

[13] M. H. Stone, The theory of representations for Boolean algebras, T. Am. Math. Soc. 40 (1936), 37-111. DOI: 10.1090/S0002-9947-1936-1501865-8

[14] Z. Vaziry, S. B. Nimse and D. Leseberg, B-nearness on Boolean frames, Rostock. Math. Kolloq. 67 (2012), 3-19.

Zohreh Vaziry, Department of Mathematics, University of Pune, Pune-411007, India e-mail: z_m_vaziry@yahoo.co.in

Dieter Leseberg, Department of Mathematics and Computer Science, Free University of Berlin, Arnimallee 14, 14195 Berlin, Germany. Chemistry Library, Technical University of Braunschweig, Hagenring 30, 38106 Braunschweig, Germany

e-mail: d.leseberg@tu-bs.de 
